

\title{
INDUCTIVE ADDER DEVELOPMENT
}

May 1989

\begin{abstract}
R. Bruce Miller John Bayless/PSI Ben B. Davis
\end{abstract}

Prepared for

Los Alamos National Laboratory

Post Office Box 990

Los Alamos, New Mexico 87545

Contract \#9-X68-5201R-1

\section{Prepared by}

TITAN/SPECTRON DEVELOPMENT IABORATORIES

2017 Yale Boulevard SE

Albuquerque, NM 87185

Mailing Address: Post Office Box 4399 Albuquerque, NM 87196

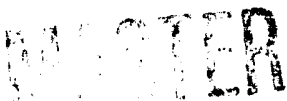


ABSTRACT

TITAN has successfully developed an Inductive Adder for use with a Hewlett-Packard Model $43734 \mathrm{~A}$ Marx pulser. The unit provides an $800 \mathrm{kV}$ peak output pulse to a modified HP 5081-9551 $1 \mathrm{MV}$ x-ray tube. The tube fits into the adder unit, and can thus be remotely operated. It delivers a peak on-axis dose of $35 \mathrm{mR}$ at a one meter distance. Supporting radiography analyses, a description of the inductive adder approach and construction, and detailed test data are presented. 
LIST OE FIGURES.

I

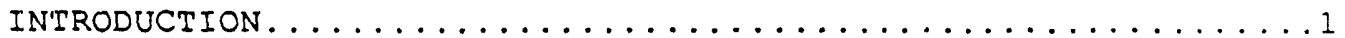


LIST OF EIGURES

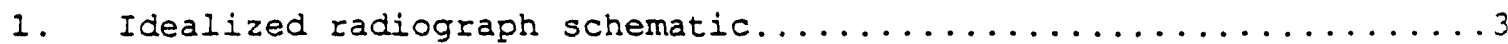

2. The collisional and radiational energy losses of

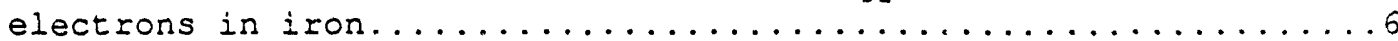

3. An example collimation of the photon beam for

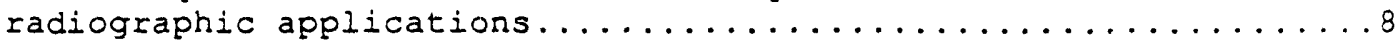

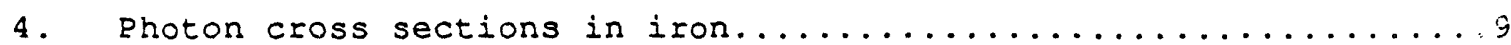

5. Standard dual remote tube head radiography

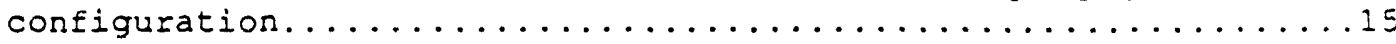

6. Desired inductive adder radiography configuration.........15

7. Schematic operation of an induction acceleration cavity................................

8. Schematic diagram of the Inductive Adder..............18

9. Layout drawing of the Inductive Adder.................20

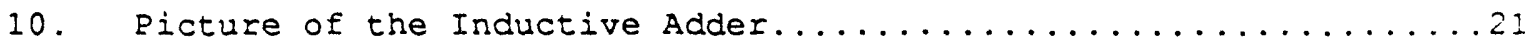

11. Picture of Adder with the $x$-ray tube removed............22

12. Picture of induction core assembly and core reset

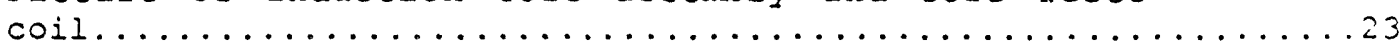

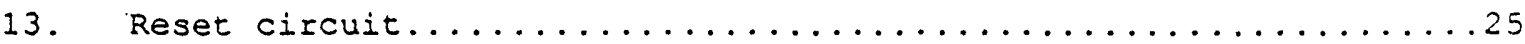

14. Equivalent circuit of the Inductive Adder..............26

15. Dependance of load voltage on load impedance............27

16. Current through the Inductive Adder. The maximum current in the first peak is $3.5 \mathrm{kA}$ the time scale is $50 \mathrm{~ns}$ per division. (Standard HP $1 \mathrm{MV}, 6 \mathrm{kA} x$-ray

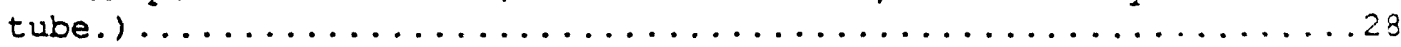

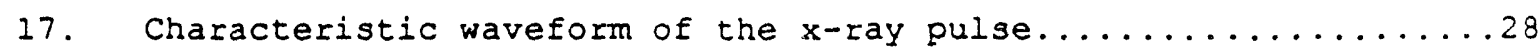

18. Load impedance of the standard $1 \mathrm{MV} x$-ray tube as a

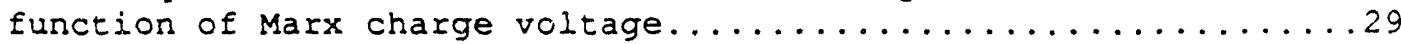

19. Adder output voltage for the standard $1 \mathrm{MV} x$-ray tube

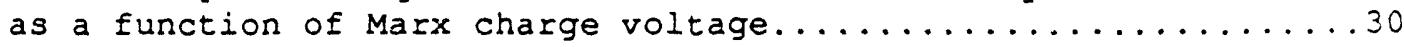

20. $x$-ray dose at one meter vs Marx charge voltage

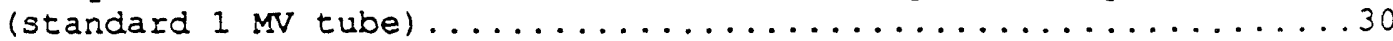


21. Current through the Inductive Adder. The maximum current in the first peak is 2. " $\mathrm{kA}$. (Modified high

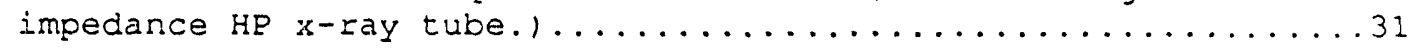

22. Load impedance of the modified high impedance $1 \mathrm{MV}$ tube as a function of Marx charge voltage.................

23. Adder output voltage for the modified high impedance 1 $M V$ tube as a function of Marx charge voltage.................

24. Variation of $x$-ray dose at $50 \mathrm{~cm}$ from the tube as a function of steel penetration thickness for several

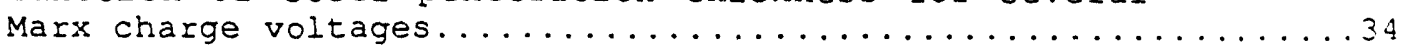

25. Configuration of radiography tests........................

26. Radiography for shot \#3. No image intensifier screen.

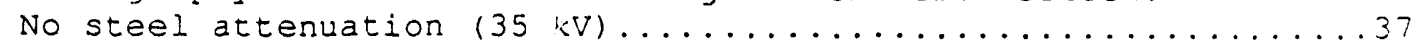

27. Radiograph for shot \#4. NDT-2 intensifier screen. No

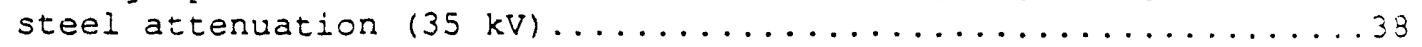

28. Radiograph for shot \#6. NDT-2 intensifier screen.

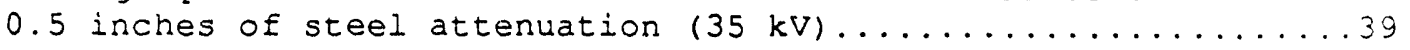

29. Radiograph for shot \#26. QUANTA intensifier screen.

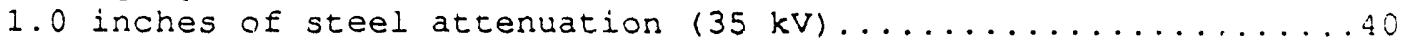

30. Radiograph for shot \#33. QUANTA intensifier screen. 1.5 inches of steel attenuation $(35 \mathrm{kV}) \ldots \ldots \ldots \ldots \ldots \ldots \ldots \ldots$

31. Radiograph for shot \#34. QUANTA intensifier screen.

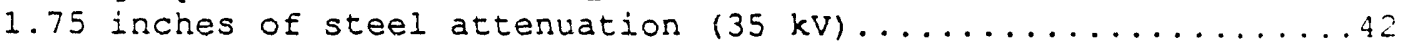


A detailed understanding of the key physics issues involved in many complex hydrodynamic processes often necessitates the use of flash $x$-radiography. In this technique energetic photons generated by electron bremsstrahlung are used to take short-duration $x-r a y$ "snapshots" at particular times during the hydrodynamic event. The time duration of the $x$-ray pulse is thus chosen to be short in comparison with the characteristic motion time of the event; exposure times of a few tens of nanoseconds are typical. In addition, it is often necessary to view the phenomena of interest through thick absorbing materials. Finally, many events of interest are very violent, necessitating additional shields and substantial standoff distances for both the $x$-ray source and the recording medium (usually an $x$-ray film cassette). As a result, relatively high voltages (typically several hundred kilovolts) are required to produce useful x-ray intensities.

The current state-of-the-art in flash radiography is exemplified by the Model 43700 Flash X-Ray Systems manufactured by Hewlett-Packard (HP). HP advertises a choice of $150 \mathrm{kV}, 300 \mathrm{kV}, 450 \mathrm{kV}, 1 \mathrm{MV}$, and 2.3 MV $x$-ray pulse generators for providing single, sub-microsecond exposures, from single or mixiple-channel (x-ray sources) configurations, using single or dual $x$-ray tube heads. They advertise more than 1600 channels in use by over 200 facilities worldwide.

As an example, consider the Model 43734A, which provides a single $25 \mathrm{~ns}$ pulse of $450 \mathrm{kV}$ x-rays. This device uses a Marx generator co supply a nominal $6 \mathrm{kA}$ current pulse to a field emission $x$-ray tube. The nominal $x$-ray dose at one meter from the tube is $20 \mathrm{mR}$. It can be operated with the $x$-ray tube in the pulser, or in a remote tube head, or with two tubes in twe remote heads ( $3 \mathrm{kA}$ to each head). In the latter configuration, both tubes are fired simultaneously by the same pulser.

The remote tube head feature means that the $x$-ray tube itself can be placed near the event, while the Marx pulser can be placed behind protective shielding. In this case hhe high voitage pulse is delivered 
to the tube head via a high voltage cable. Unfortunately, this attractive feature is not available for the higher voltage pulsers (such as the $1 \mathrm{MV}$ HP $43710 \mathrm{~A}$ ) because of cable breakdown difficulties.

For a particular hydrodynamic problem of interest to Los Alamos National Laboratory it was desired to produce high energy $x$-rays $1 \geq 800$ $\mathrm{kV})$, but to do so using a remote tube head. TITAN's solution for this problem was the development of an inductive adder. Specifically, an $H P$ Model 43734A pulser was operated in a dual remote tube head configuration. However, both high voltage output cables were inserted into an inductive isolator device which allowed the sum of the two voltage pulses to be delivered to the nominal $1 \mathrm{MV} x$-ray tube used in the HP $43710 \mathrm{~A}$ pulser. This final report summarizes the physics of bremsstrahlung radiography, operation of the inductive adder, and the results of $\mathrm{x}$-radiography experiments using stationary targets performed prior to the installation of the device at Los Alamos.

II. IDEAIIZED RADIOGRAPHY ANALYSIS ${ }^{1}$

Consider the schematic geometry of Figure 1. A uniform pulse of monoenergetic photons of intensity $I_{0}$ is normally incident on a homogeneous slab of total thickness $L$ which has an internal cubical void of thickness $\Delta I$. It is assumed that the photons undergo only absorption events in the material. If the atomic density in the slab is $n$, and the photon absorption cross section per atom is $\sigma$, then the change in the photon flux in the infinitesimal thickness $\mathrm{dx}$ at depth $\mathrm{x}$ is given by $\mathrm{dI}$ $=-\sigma n I d x$. The depth-dependent photon flux can be obtained by integration, and is simply

$$
I(x)=I_{0} e^{-\sigma n x}
$$

From Eq. (1), the average photon flux which reaches the detector plane after having passed through the total slab thickness $I$ is just

$$
I_{a}=I_{0} e^{-\sigma n L},
$$


while the photon flux reaching the detector plane after having passed through the region of the void is

$$
I_{v}=I_{0} e^{-\sigma n(I-\Delta L)}
$$

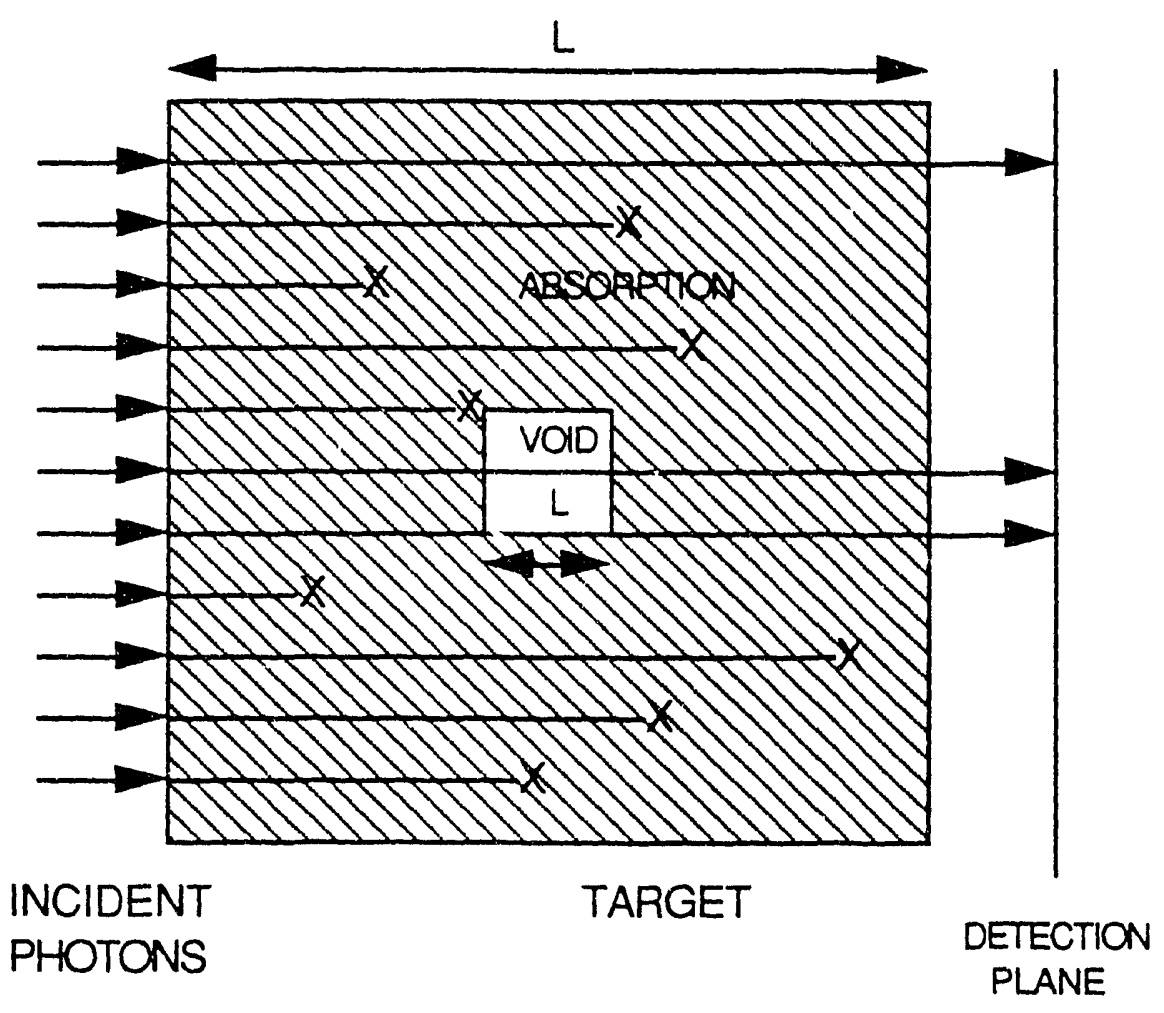

Eigure 1. Idealized radiograph schematic.

Assuming the detector records all photons reaching it, the presence of the void is indicated by the contrast in the photon flux, or

$$
\begin{aligned}
& C=\left(I_{v}-I_{a}\right) / I_{a} \\
& =e^{\sigma n \Delta I-1} \\
& =\text { on } \Delta I
\end{aligned}
$$

for small voids. 
Eq. (2) suggests that the contrast can be improved by choosing a photon energy for which the absorption cross section is large; however, if the attenuation is too large, the statistical variation in the average transmitted flux will cause a loss of contrast.

The effective signal-to-noise ratio is defined by

$$
S / N=\left(I_{v}-I_{a}\right) / \delta I_{a}
$$

where $\delta I_{a}=\left(I_{a}\right)^{1 / 2}$ is the standard deviation of the average unscattered photon flux. For this problem we have

$$
S / N=\sigma n \Delta I \quad\left(I_{0} e^{-\sigma n L}\right)^{I / 2}
$$

The maximum value attained by Eq. (3) is

$$
(S / N)_{\max }=(2 / e)(\Delta L / L) I_{0} 1 / 2
$$

which occurs when $\sigma_{n}=2 / \mathrm{L}$, i.e., when the thickness of the object is equal to two mean free paths. Under this condition the contrast is given by $\mathrm{C}=2 \Delta \mathrm{L} / \mathrm{L}$.

Of course, this description is far too simplistic. In practice there are several factors which can considerably complicate this picture:

(1) If the photons are generated by electron bremsstrahlung, then the spectrum is far from monoenergetic.

(2) The normally-incident, parallel photon beam is generally approximated by a point source with a large converter-object distance.

(3) The removal of primary photons is generally not absolute; scattering processes and secondary radiation generation can increase the noise level at the detection plane.

(4) The detection and recording of the transmitted photon flux can be inefficient. 
(5) Eor fast hydrodynamic events, short photon pulses are necessary to prevent motion blur.

In order to decrease the negative influence of several of these factors it is necessary to have a good understanding of the several physical processes involved in the generation, transmission, and detection of bremsstrahlung $x$-rays. We briefly review these processes in the next sectior.

\section{PHYSICS OF BREMSSTRAHLUNG RADIOGRAPHY}

A. Eremstrahlung Generation

The important energy loss mechanisms for energetic electrons are collisional excitation and ionization processes, and radiative interactions (bremsstrahlung). The ionization energy loss per unit length scales linearly with the atomic density and atomic number of the target atoms, and weakly (logarithmically) with the beam kinetic energy. A useful approximate (empirical) expression is

$$
\rho^{-1}(d E / d x)_{\text {ior }}=1.5 \mathrm{MeV} \mathrm{cm}^{2} / \mathrm{g}
$$

where $\rho$ is the density of the target material. In contrast, the energy loss due to bremsstrahlung is proportional to the square of the atomic number and increases nearly linearly with the kinetic energy of the incident electron. The ratio of the radiative loss to the ionization loss is given approximately by ${ }^{2}$

$$
\frac{(d E / d x) r a d}{(d E / d x) \text { ion }} \sim \frac{E(M e V) Z}{800}
$$

Hence, the range of a $20 \mathrm{MeV}$ electron in aluminum is about $4 \mathrm{~cm}$, while in tungsten the range is about $0.5 \mathrm{~cm}$. In the first case almost all the energy is deposited locally (only ionization), while in the latter case there are significant radiation losses. The energy dependence of these 
processes is emphasized in Figure 2 which exhibits the collisional and radiative stopping powers in iron as a function of energy.

The bremsstrahlung angular distribution and yield for thick targets depend not only on the initial beam description at the target but also on the further transport of the beam into the target. Because the ratio of $\mathrm{e} / \mathrm{m}$ is so high for electrons the ionization and radiative collisions cause significant deflections of the incident beam. The energy of the emitted photon can vary from zero up to the kinetic energy of the incilent electron, while the angular distribution of the radiation becomes narrower with increasing energy. For relativistic electrons the average angle between the direction of the incident electron and the emergent photon can be approximated by $\theta=\mathrm{m}_{0} c^{2} / \mathrm{E}$.

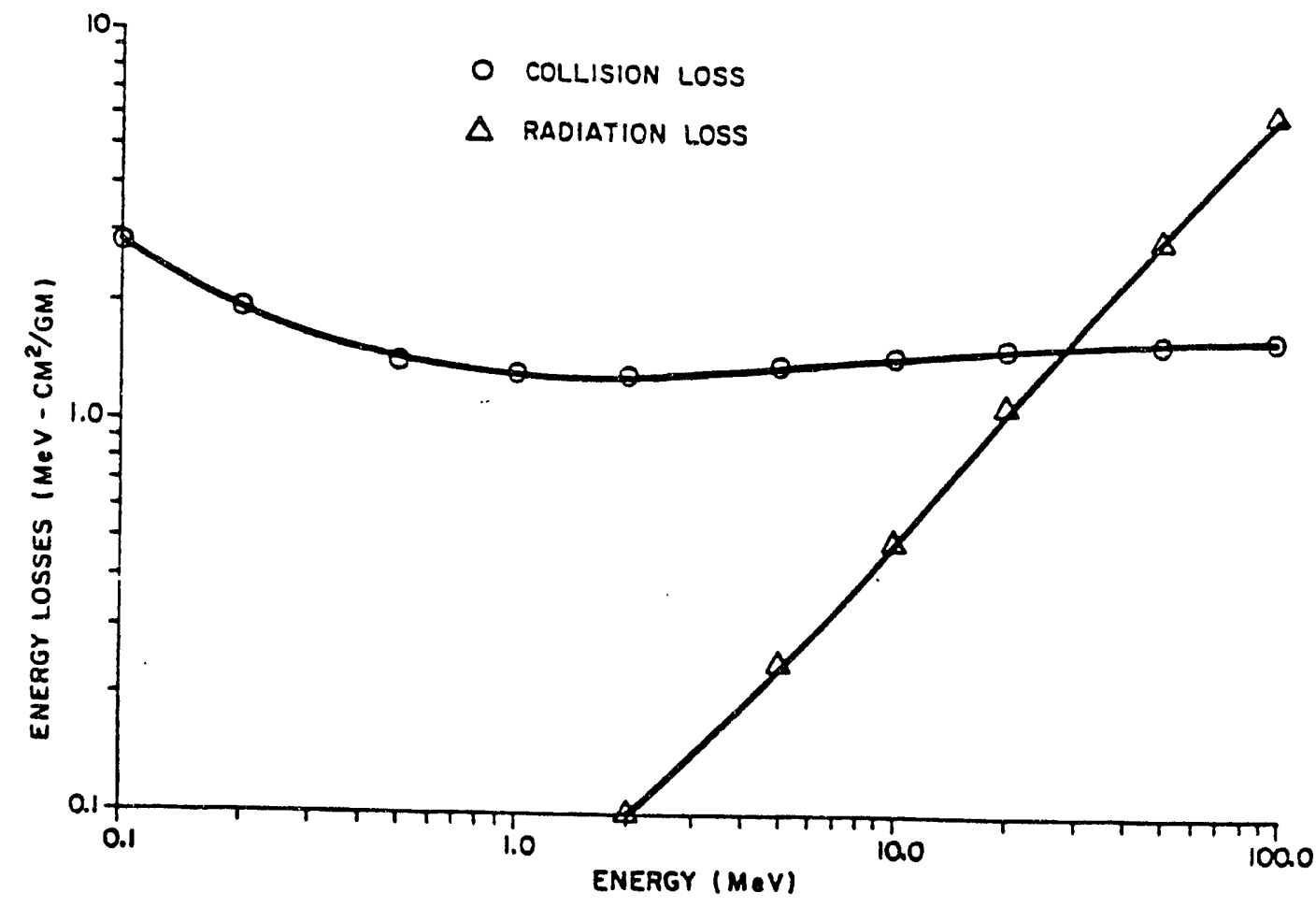

Figure 2. The collisional and radiational energy losses of electrons 
An approximate analytical expression which describes the differential photon flux density is ${ }^{3}$

$$
d \psi / d E_{p}=\varepsilon_{p}(E) K e^{\alpha E p}
$$

where $E$ and $E_{p}$ are the energies of the incident electron and emerging photon, the exponent $\alpha E_{p}$ has an average value of $\left(-3.75 E_{p} / E\right)$, and $K$ is a numerical constant. The differential intensity is then given by

$$
d I / d E=\varepsilon_{p}(E) K E_{p} e^{\alpha E p}
$$

Over the energy interval 2-20 MeV, a good assumption is that the efficiency $\varepsilon_{p}(E)$ of bremsstrahlung production varies as $E^{0.8}$. Inserting the numerical value for the constant $K$ and integrating over all photon energies then gives the total intensity at one meter from the source as ${ }^{3}$

$$
I_{t}=2.3 \times 1012 \mathrm{E}^{2.8}\left(\mathrm{MeV} / \mathrm{cm}^{2}-\mathrm{C}\right)
$$

The dose rate for photons of a few MeV is approximately given by

$$
D=5.2 \times 10^{-10} I_{t} \text { (Roentgens/sec) }
$$

Substituting for $I_{t}$ then gives the dose per coulomb at one meter from the converter as

$$
D / Q=1.2 \times 10^{3} E^{2.8} \text { (Roentgens/Coulomb) }
$$

As a comparison, Martin has proposed an empirical relation of

$$
D / Q=1.1 \times 10^{3} E^{2.8} \quad(R / C)
$$

based on an analysis of PHERMEX data. ${ }^{4}$ The agreement is excellent considering the numerous app:oximations and assumptions involved.

There are several important practical radiographic considerations, in addition to the generation of the largest quantity of useful photons. 
For example, photons emitted from the $x$-ray tube at large angles with respect to the target direction are not useful for image formation, and can contribute to a loss of contrast as the result of scatter from floors and walls. This undesirable effect can be reduced by inserting high-z collimation between the bremsstrahlung crnverter and the object to be radiographed, as shown schematically in Figure 3.5

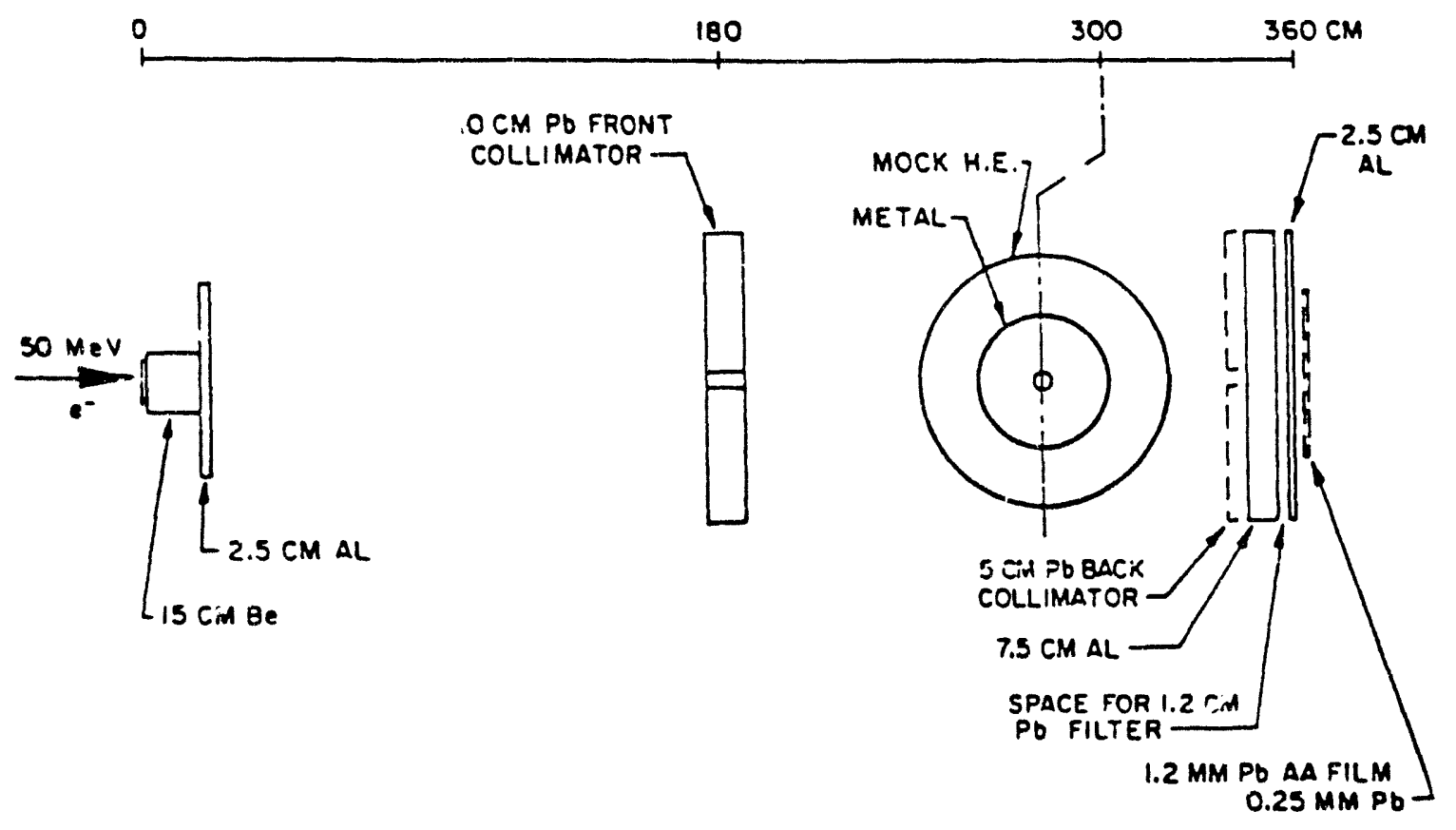

Figure 3. An example collimation of the photon beam for radiographic applications.

Also, the convezter-target distance lot for a given resolution requirement (we assume $0.4 \Delta L$ ) is essentially determined by the beam spot size d at the converter and the distance from the target to the $x-$ ray detection plane. From simple geometrical considerations

$$
I_{c t}=I_{t d} \frac{d}{0.4 \Delta L}
$$


Hence, $I_{c t} \approx 112 \mathrm{~cm}$ is required to resolve $1 \mathrm{~mm}$ features with a 15 $\mathrm{cm}$ target-detection plane distance and a beam spot size diameter of 3 $\mathrm{mm}$.

B. Passage of Energetic Photons Through Matter

The important mechanisms by which energetic photons interact with matter include (1) the photoelectric effect, (2) Compton scattering, and (3) pair production. ${ }^{2}$ Each of these processes is fairly comples, and is accompanied by secondary effects such as fluorescence radiation in (1), the emission of recoil electrons in (2) and the subsequent annihilation of positrons in (3). The three processes have different relative importance in different spectral regions, depending on the atomic number of the absorber, as emphasized by the photon cross section data for iisn presented in Eigure 4.6

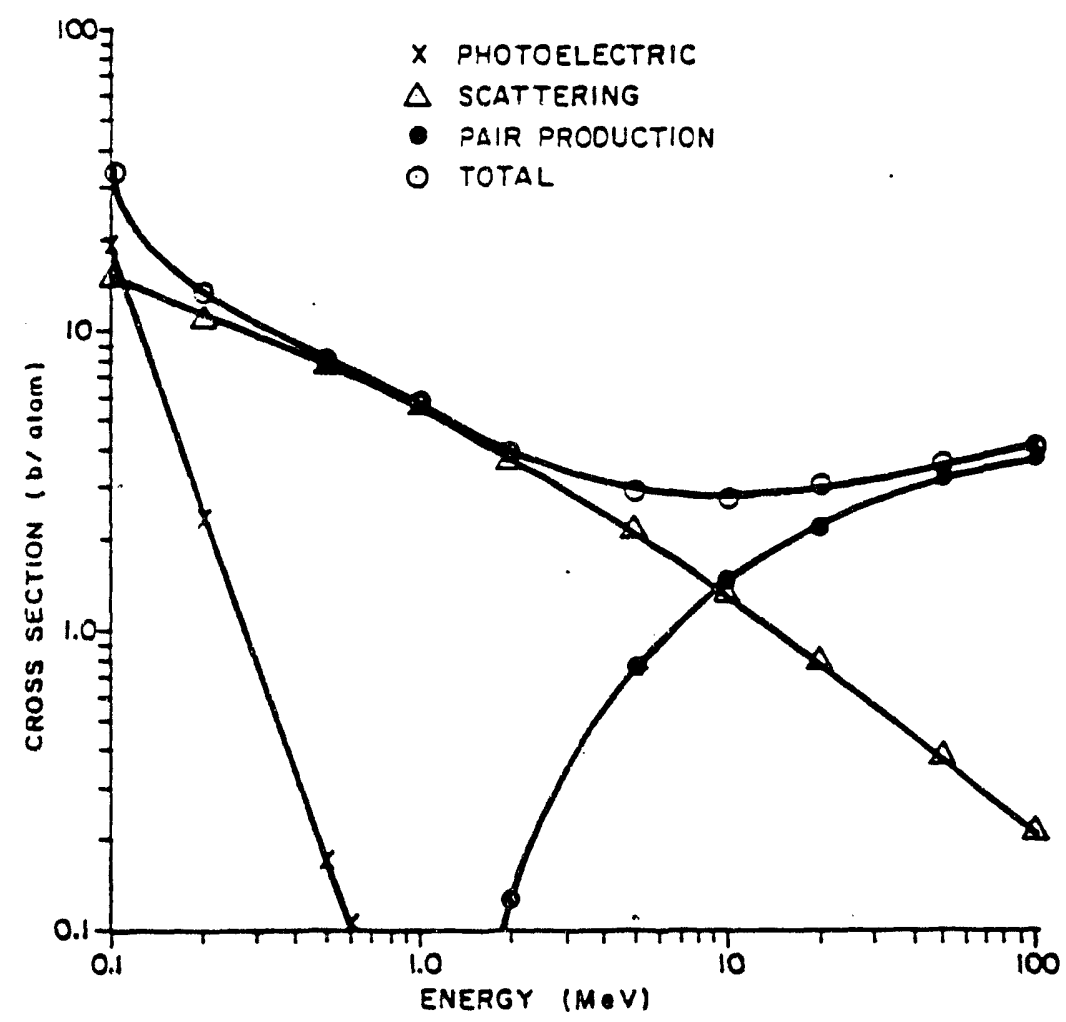

Eigure 4. Photon cross sections in iron. 
In photoelectric absorption the energy of a photon is absorbed by an inner atomic shell electron, which is emitted with a resultant energy of $E=h v-E_{b}$, where $E_{b}$ is the binding energy. Filling the vacancy left by the ejected electron can then produce fluorescent radiation as well as additional Auger electrons. The photoelectic process is dominant at low photon energies.

The scattering of low energy photons is adequately de cribed by Thomson's classical theory. However, for photon energies comparable with the rest mass energy of an electron ( $0.511 \mathrm{MeV})$, conservation of energy and momentum require the energy of the scattered photon to be less than that of the incident photon, with the resultant kinetic energy difference being taken up by the recoil electron. Compton scattering is usually the dominant process for intermediate photon energies.

Finally, in pair production an energetic photon can be transformed into an electron-positron pair. The threshold energy in the center-ofmass system for this materialization process to occur is simply twice the rest mass energy of an electron. Annihilation of the positron subsequently results in the creation of two photons of $0.511 \mathrm{MeV}$ each. Pair production is usually dominant at high photon energies.

Having reviewed the important photon interaction mechanisms, we now describe the transport of the bremsstrahlung through the radiographed object. From the simple example of Figure 2 , it is apparent that the information which contains the structural details of the radiographed object is carried by the unscattered, unabsorbed photon flux reaching the detector plane. Because of the energy dependence of the bremsstrahlung, the equivalent expression for those unscattered photons which do not pass through the void region is an integral over the bremsstrahlung spectrum $S\left(E_{p}\right)$, using the energy dependent photon cross section.

$$
I_{a}=\int S\left(E_{p}\right) e^{-n \sigma}\left(E_{p}\right) L d E_{p}
$$


while the equivalent expression for the photons passing through the void is

$$
I_{v}=\int S\left(E_{p}\right) e^{-n} \sigma(E p)(L-\Delta L) d E_{p}
$$

Because of the secondary processes described above, the interaction of the primary bremsstrahlung photons will result in a photon-electron cascade. This process, which is usually modeled through use of a build-up factor, can be quite important because some of the resulting photons will reach the detection plane without adding to the information content of the image. The build-up factor is usually defined as the ratio of the sum of the scattered and unscattered photons to the number of unscattered photons, i.e.,

$$
B\left(E_{p}, L\right)=\left(N_{S}\left(E_{p}, L\right)+N_{U}\left(E_{p}, L\right)\right) / N_{U}\left(E_{p}, L\right)
$$

It is usually necessary to use a Monte Carlo transport code, such as the ITS series ${ }^{7}$ developed by Halblieb or LANL's MCNPE, to estimate the build-up factors for each geometry of interest.

If the volume of the void is small compared to the total volume of the object, then most of the scattered photons arriving at the detection plane in the region of the void image will have been generated in the ambient material. With this assumption, and our previous definition, the contrast in the presence of build-up is approximately given by

$$
C=\int\left(\left(\left(N_{u}\left(E_{p}\right)\right)_{v} /\left(N_{u}\left(E_{p}\right)\right)_{a}\right)-1\right) / B\left(E_{p}\right) d E_{p}
$$

The decrease in contrast for large build-up factors, which would be expected for penetration through thick objects, is apparent. In addition, it is easy to show that the signal-to-noise ratio is also decreased by the square root of the build-up factor. 


\section{RADIOGRAPHIC PULSER FARAMETER CONSIDERATIONS}

In the design of a high voltage source for a radiographic system, two of the most important factors are the choice of electron energy and the total charge that must be delivered. Guided by the results of the preceding sections, we have developed an approximate analysis for estimating the required pulser voltage and current.

Taking into account the photon deep-penetration build-up factor $B$, and the detector efficiency $\eta$, we model the contrast $C$ and the $S / N$ ratio of a radiographic image as

$$
\begin{aligned}
& C=\frac{\eta_{u}\left(I_{v}-I_{a}\right)}{\left[(B-1) \eta_{s}+\eta_{u}\right] I_{a}} \\
& S / N=\frac{\eta_{u}\left(I_{v}-I_{a}\right)}{\left\{\left[(B-1) \eta_{s}+\eta_{u}\right] I_{a}\right)^{1 / 2}}
\end{aligned}
$$

where $I_{a}$ is the average unscattered photon flux that has passed through the complete target thickness, and $I_{V}$ is the unscattered photon flux that has passed through a perturbation in the target (a void, for example). The $u$ and $s$ subscripts are used to denote the detector efficiencies for the unscattered and scattered photon fluxes. It is assumed that all photons have the average energy $\bar{E}_{p}$ of the bremsstrahlung spectrum $S\left(E_{p}\right)$, as defined by

$$
\bar{E}_{p}=\frac{\int E_{p} S\left(E_{p}\right) d E_{p}}{\int S\left(E_{p}\right) d E_{p}}
$$

In general, the scattered flux spectrum will be somewhat softer than that of the unscattered flux, i.e., $\eta_{u}<\eta_{g}$.

To illustrate the process, consider the problem of resolving a two millimeter crack in a $2.5 \mathrm{~cm}$-thick section of iron. The optimum $S / N$ ratio occurs when the thickness $L$ of the object is about twice the photon mean free path. (We use this result, recognizing that the effect 
of the variation in build-up factor with object thickness is being ignored.) The desired cross section is about 9.4 barn/atom for this thickness of iron. Referring to the iron cross section data in Figure 4, the desired photon energy is about $0.35 \mathrm{MeV}$. In this energy range the average photon energy of the bremsstrahlung spectrum is about $33 \frac{z}{\circ}$ of the electron energy, implying a desired electron energy of about 1 MeV. For this object thickness and energy range, the build-up factor will be approximately 2.4. Assuming that $\eta_{\mathrm{S}}=\eta_{\mathrm{u}} \approx 0.2$, then from Eq. (16)

$$
C=2 \Delta \mathrm{I} / \mathrm{BL}=6.7 \frac{8}{8}
$$

where $\Delta I=2 \mathrm{~mm}$ is the size of the crack.

Eq. (17) is used to determine the required accelerator current, which becomes under our previous assumptions

$$
S / N=(2 / e)(\Delta L / L)\left(\eta I_{0} / B\right)^{0.5}=1.7 \times 10^{-2}\left(I_{0}\right)^{0.5}
$$

To achieve a $\mathrm{S} / \mathrm{N}$ ratio of $10, I_{0}=3.5 \times 10^{5}$ photons over the area, $A_{2}$, of a resolution cell. Since we are interested in $2 \mathrm{~mm}$ features, we assume $A_{I}=(0.2 \Delta I)^{2}=1.6 \times 10^{-3} \mathrm{~cm}^{2}$. Hence, $I_{0}=2.15 \times 10^{3}$ photons $/ \mathrm{cm}^{2}$, or

$$
I_{t}=7.5 \times 10^{7} \mathrm{MeV} / \mathrm{cm}^{2}
$$

assuming an average photon energy of $0.35 \mathrm{MeV}$. Using $\mathrm{E}=1 \mathrm{MeV}$ in Eq. (8) gives a required accelerated charge of $33 \mu \mathrm{C}$. The average required beam current is about $1.3 \mathrm{kA}$ for a nominal 25 ns voltage pulse, and the radiation dose at one meter from the $x$-ray tube would be about $36 \mathrm{mR}$.

\section{INDUCTIVE ADDER APPROACH}

The Los Alamos National Laboratory requires a compact, lightweight, flash radiograph machine capable of stopping the motion of small metallic shards in flight. (The shards could be as small as a few millimeters in physical dimension.) A high signai-to-noise ratio is 
necessary, even though the radiograph might have to be taken through up to an inch of steel armoring. Remote tube head operation is also desired.

Based on the calculations of the preceeding section, the radiography machine should thus be capable of supplying $30-40 \mathrm{mR}$ at one meter from the $x$-ray tube, and should operate near 1 MV to provide adequate contrast. Because of cable breakdown difficulties, there is no off-the-shelf radiographic device with remote tube head operation that can meet these specifications. As a result, TITAN has devised the inductive adder approach to meet these radiographic machine requirements.

Consider the HP Model $43734 \mathrm{~A} 450 \mathrm{kV}$ pulser operated in the dual remote tube head configuration, as illustrated in Figure 5 . In this configuration both tubes are fired simultaneously by the same pulser, thereby providing the capability for obtaining orthogonal radiographs of rapidly moving objects, at the expense of reduced radiation dose (and penetrating power) from each tube. If it were possible to construct a device that could add the voltage pulses provided to the two separate tube heads illustrated in Figure 5, and deliver this higher voltage pulse to a suitable $x$-ray tube, then the resulting $x$-ray parameters would essentially satisfy all of Los Alamos' requirements. This new adder configuration is schematically shown in Figure 6 .

The technology that is relevant for this adder concept is that associated with linear induction accelerators. An acceleration cell of all such accelerators operates by producing voltage around a circuit enclosing a time-varying magnetic flux. Mathematically, this is described by Eq. (22), where $B$ and $E$ represent the magnetic induction and the induced electric field, and $V$ is the voltage induced around the circuit $C$ which encloses the area $A$.

$$
V=\int_{C} E \cdot d I=-(1 / C)(\partial / \partial t) \int_{S} B \cdot d A
$$




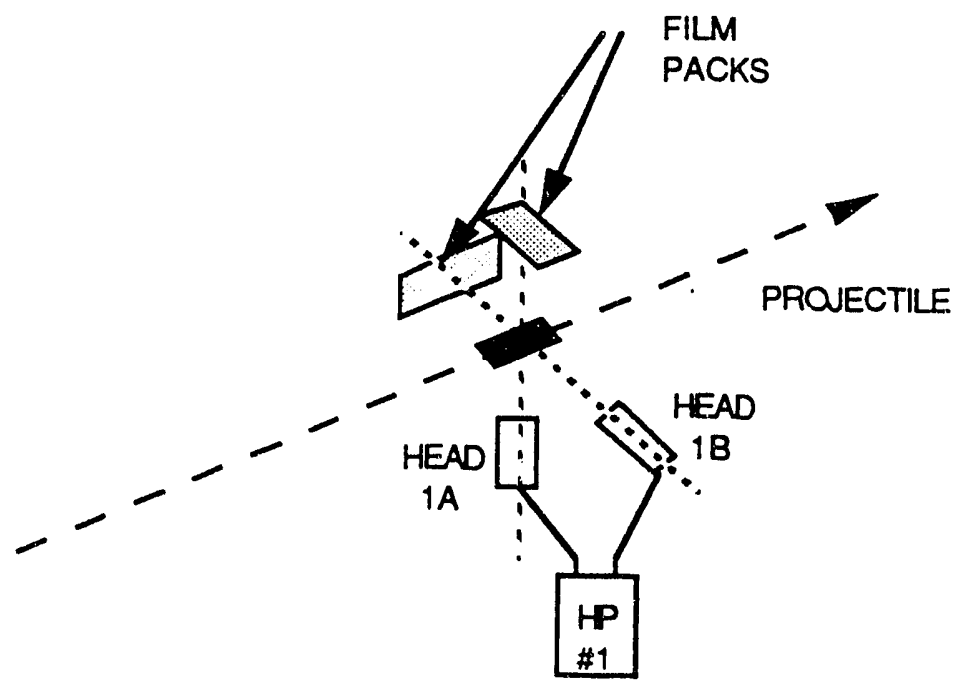

Figure 5. Standard dual remote tube head radiography configuration.

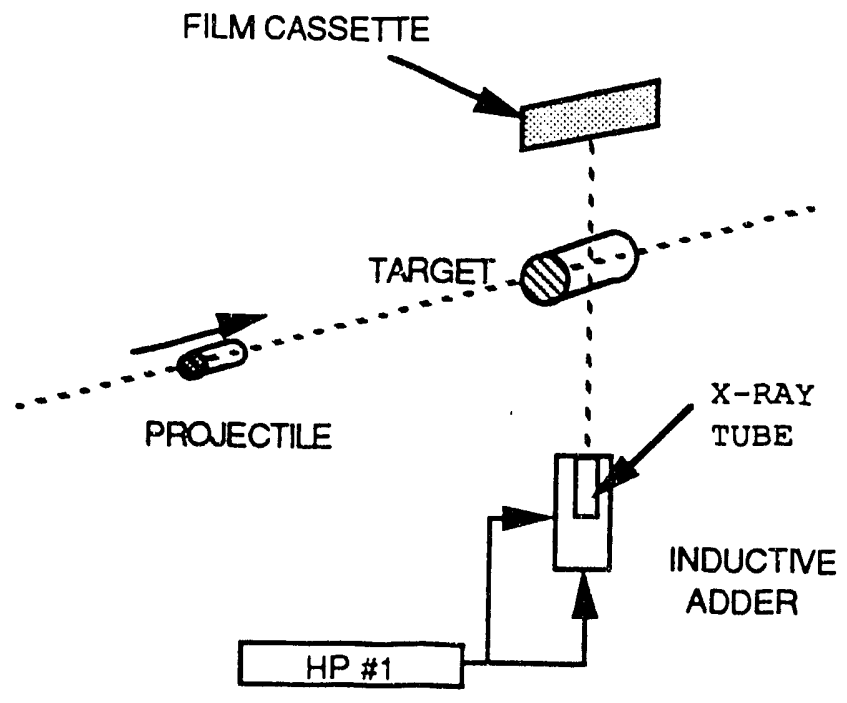

DUAL OUTPUT

Figure 6. Desired inductive adder radiography configuration. 
Time is represented by $t$, while $c$ is the speed of light. Note that Eq. (22) allows the generation of voltage by cianging either the enclosed magnetic field, or the area normal to the field lines. For example, an external circuit can be used to establish a magnetic field through a second circuit. If the current through the external circuit is timevarying, a voltage will be established at the output connections of the second circuit, i.e., this is a single turn transformer. The output voltage can be increased by inserting a core of ferromagnetic material since the B-field enclosed is increased by the magnetic permeability of the material according to $\mathrm{B}=\mu \mathrm{H}$, where $\mathrm{H}$ represents the macroscopic magnetic field, and $\mu$ is the magnetic permeability of the ferr magnetic material.

As a practical example of this approach consider the acceleration gap geometry of Eigure 7. A high voltage pulse is injected into an accelerating cavity containing an annular core of magnetic material. It is assumed that the magnetic induction pre-existing in the core is saturated at the value $+B_{0}$. Note that without the core the input pulse would be short-circuited; however, as current begins to flow around the core, the magnetic induction in the material changes until it reaches the value $-B_{0}$. During this time $T$, the accelerating voltage is effectively applied directly to the accelerating gap. From Eq. (22) these parameters are related according to $V T=D B A=2 B_{O} A$, where $A$ is the cross sectional area of the annular core. In other words, the product of the accelerating gap voltage and the pulse time is equal to the flux swing tiries the area of the annular core. Note that tre voltage does not appear external to the accelerating gap; i.e., the exterior of the accelerator remains at ground potential and the hich voltage is summed by only the beam as it propagates across the accelerating cavity. 

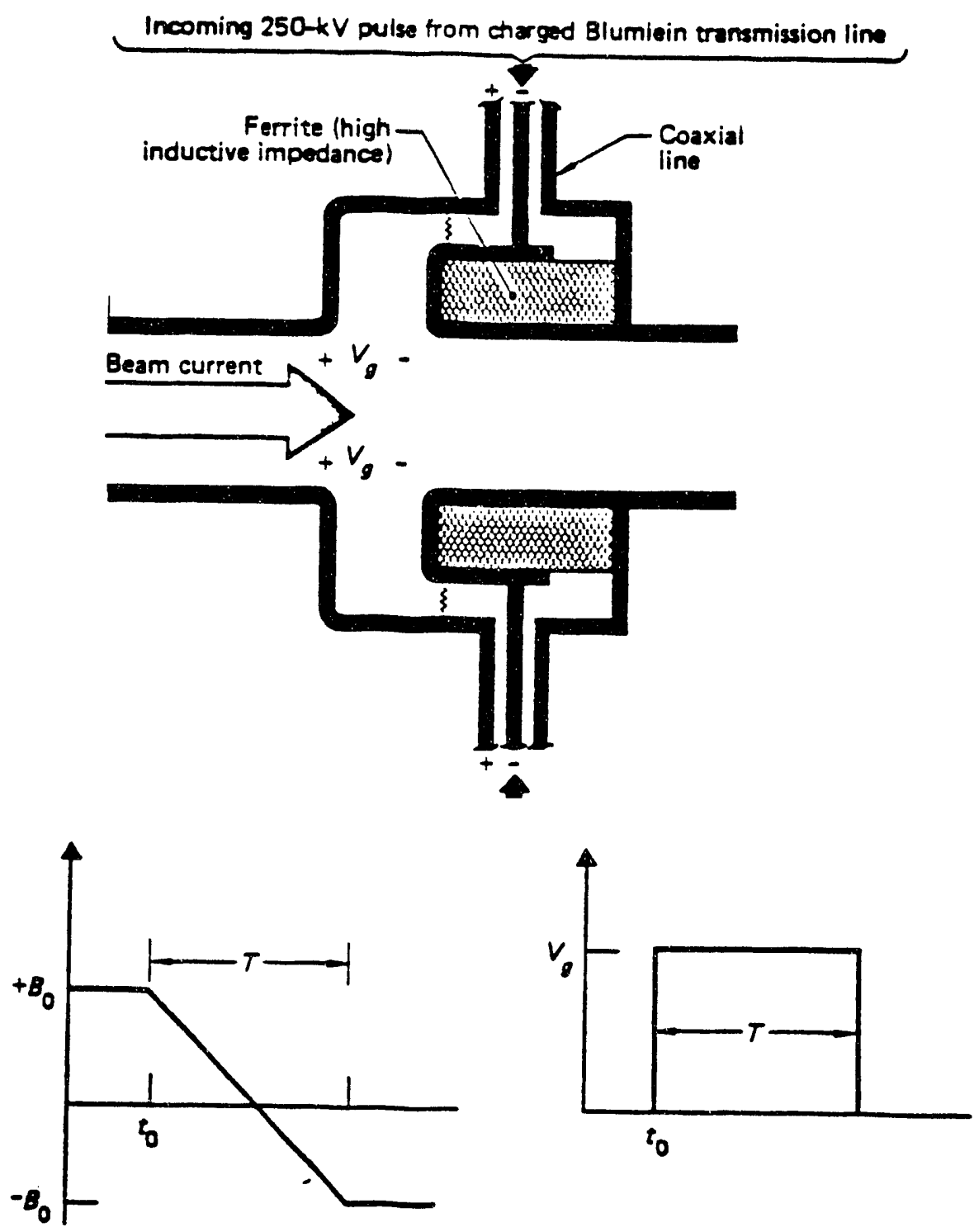

Figure 7. Schematic operation of an induction acceleration cavity. 
An alternate description of the circuit of Figure 7 is that the voltage pulse appears across the parallel combination of the impedance $\mathrm{z}_{\mathrm{b}}$ associated with the beam, and the impedance $\mathrm{z}_{\mathrm{c}}$ associated with the flow of current around the magnetic core. When the core is saturated at the level +Bo, the inductance associated with the current path around the core is very high, and $z_{C}$ is very large. Thus, the pulse energy is delivered directly to the beam. During the time $T$, however, the flow of leakage current around the core drives the core into saturation at the level $-B_{0}$. $z_{C}$ then drops to essentially zero, and the accelerating pulse is shorted.

From this discussion it is apparent that we can use this rechnique to construct the adder that is desired. Consider the schematic geometry of Figure 8. One high voltage input cable from the HP pulser is connected directly to the $x$-ray tube itself, while a second input cable is inserted into the side of the adder unit with the connections as shown. Through use of the magnetic induction cores, the two input cables are effectively connected in series across the anode-cathode gap of the $x$-ray tube, resulting in a doubling of the voltage applied to the tube.

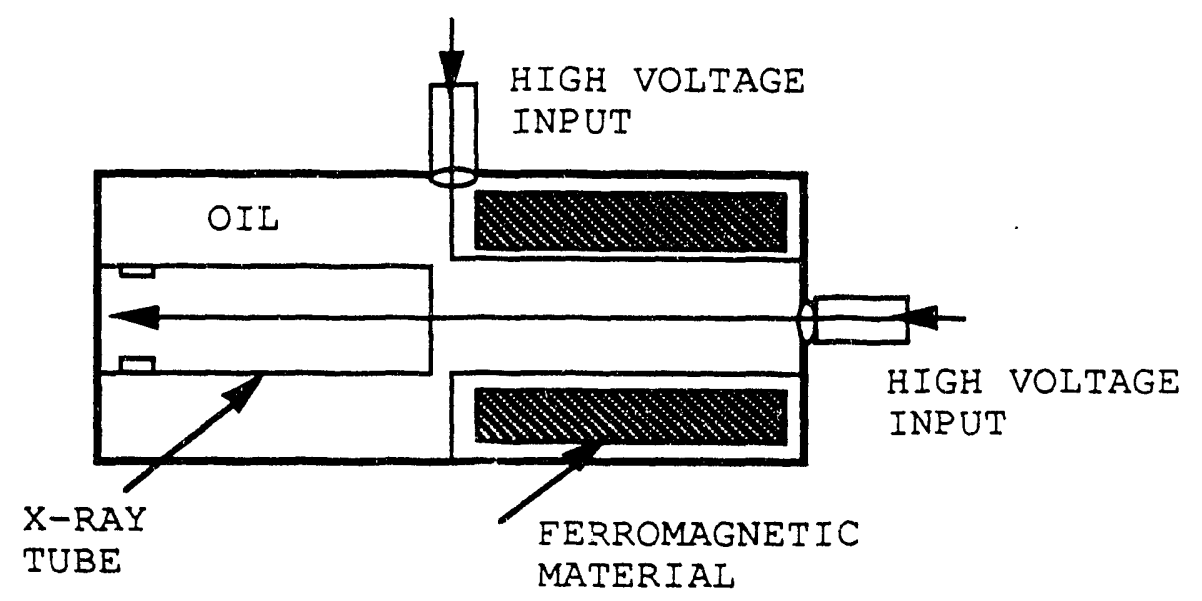

Figure 8. Schematic diagram of the Inductive Adder. 


\section{ADDER CONSTRUCTION}

The adder unit is nominally designed to combine the two hig. voltage cable outputs of in HP Model $43734 \mathrm{~A}$ pulser to deliver approximately $800-900 \mathrm{kV}$ to a $1 \mathrm{MV}$ HP $\mathrm{x}$-ray tube. The induction cell assembly and the $x$-ray tube are contained within the adder chamber which is about: 31 inches long, 13 inches in diameter, and weighs approximately
200 pounds.

A layout drawing of the Adder is shown in Figure 9, and Figures 10 through 12 show pictures of the Adder and its components.

The Adder consists of the following major components:

1. Core Assembly - Twelve 8.5 inch o.d. $x 2.5$ inch i.d. $x 1$ inch thick ferrite cores provide an inductance of $\geq 20$ mh with a volt-second product of $.014 \mathrm{~V}-\mathrm{sec}$ as required to inductively isolate the radial cable input from ground. The peak magnetization current is expected to be less than $700 \mathrm{~A}$.

2. X-ray Tube - The $1 \mathrm{MV}$ tube, which is clamped and sealed into the chamber end plate, is located near the end of the core assembly.

3. Cable Connections - One cable enters the Adder on axis and is machined to fit into the reentrant portion of the $x$-ray tube. A flexible connector is attached to the end of the cable center conductor as an aid in making good electrical contact to the tube terminal. The second cable enters the chamber from the side and plugs into the electrode that is grounded through the core assembly.

4. Reset Inductor - This inductor, which is wound on the plastic cylinder surrounding the $x$-ray tube, allows core reset current to flow on a long time scale but isolates the short high voltage pulses from the reset circuit. 


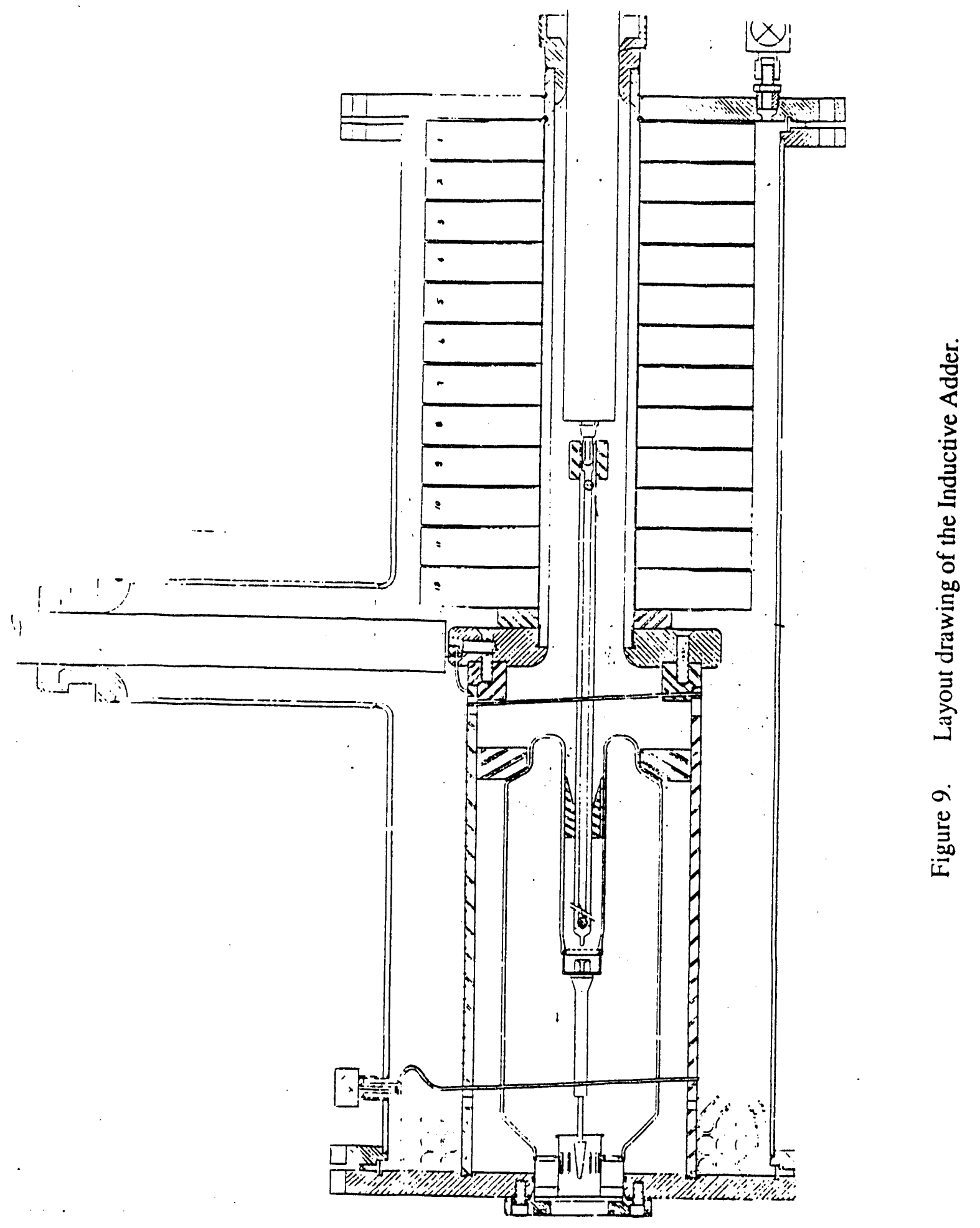




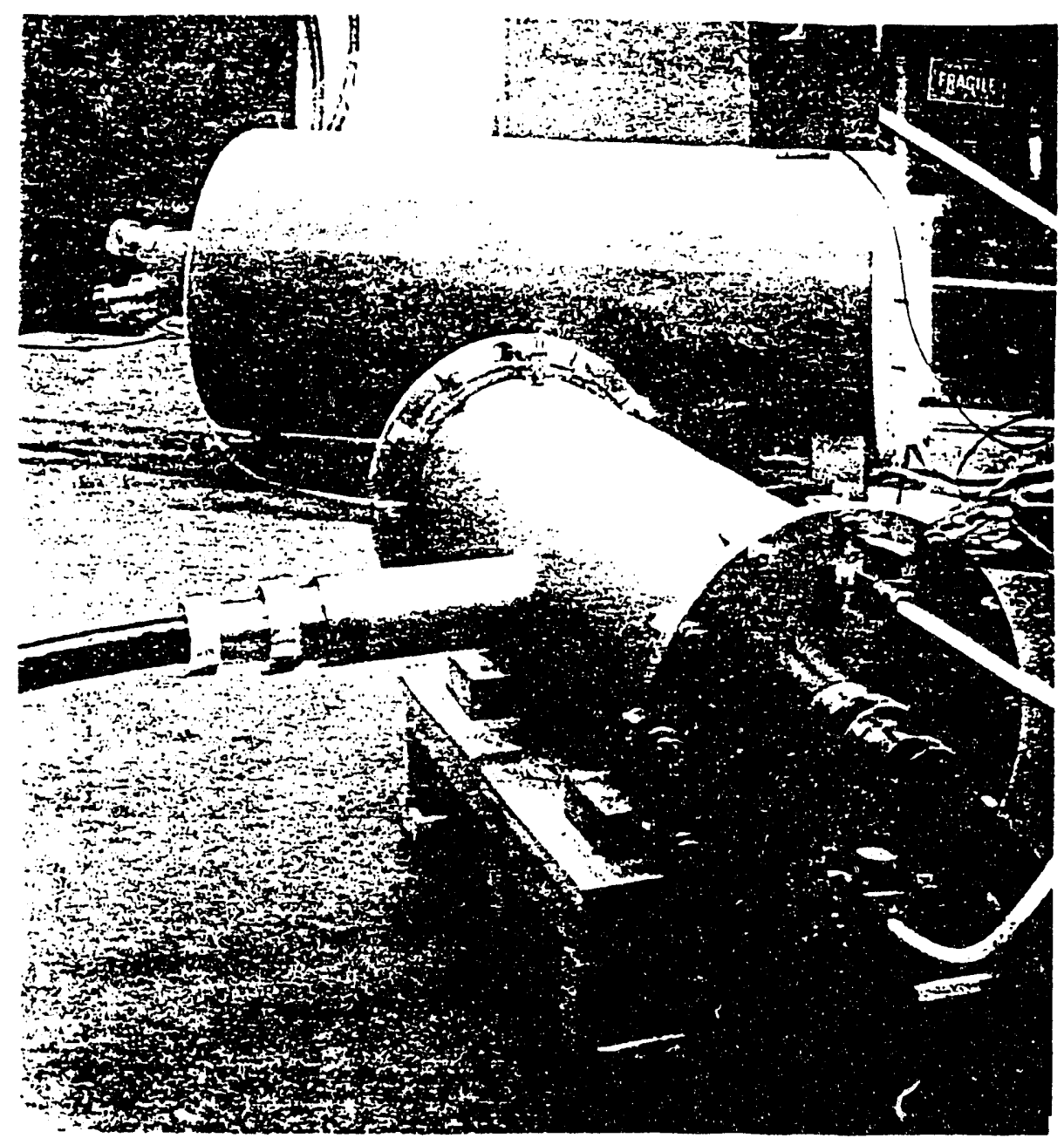

Figure 10. Picture of the Inductive Adder. 


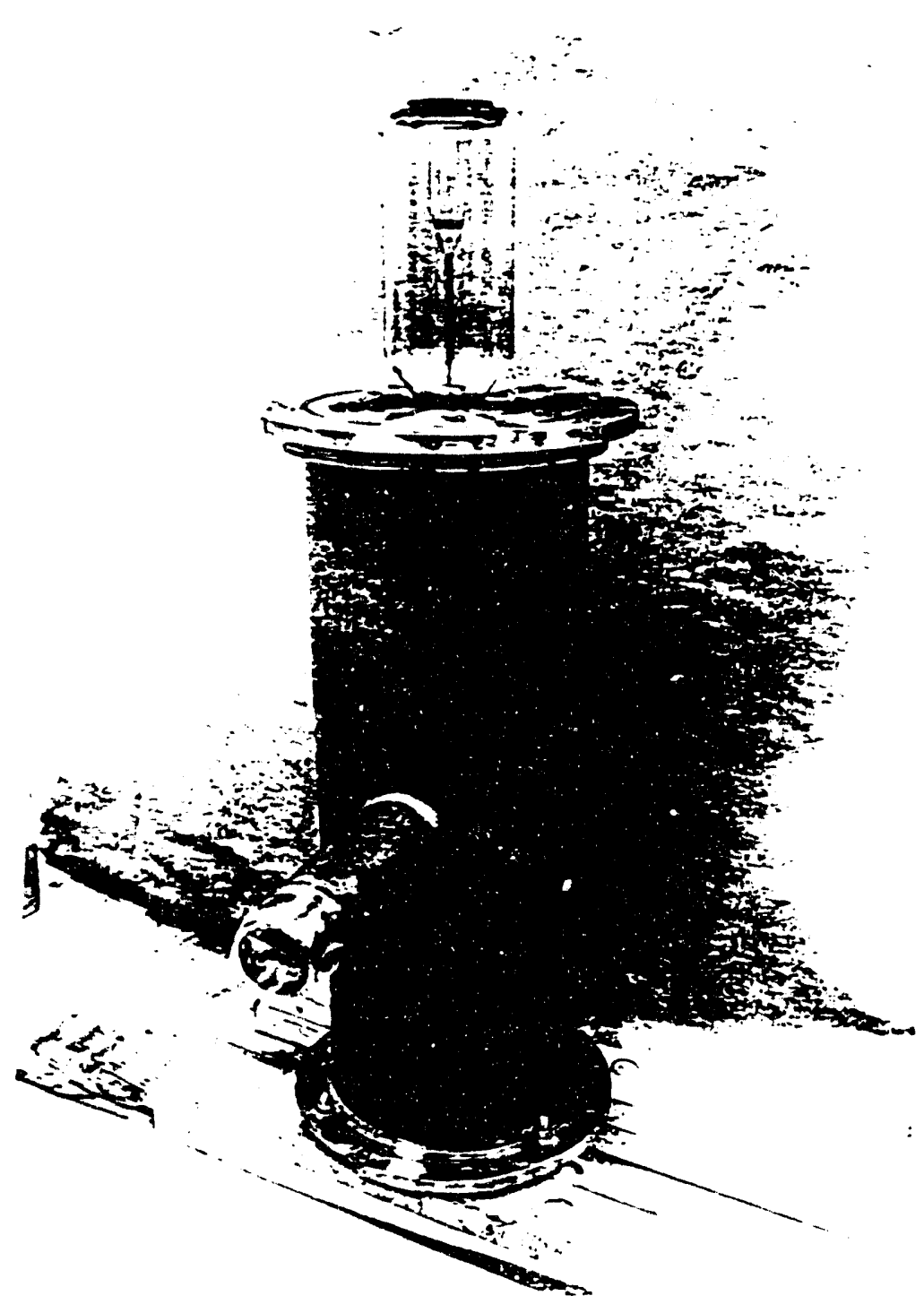

Figure 11. Piciure of Adder with rhe $x$-ray fube removed. 


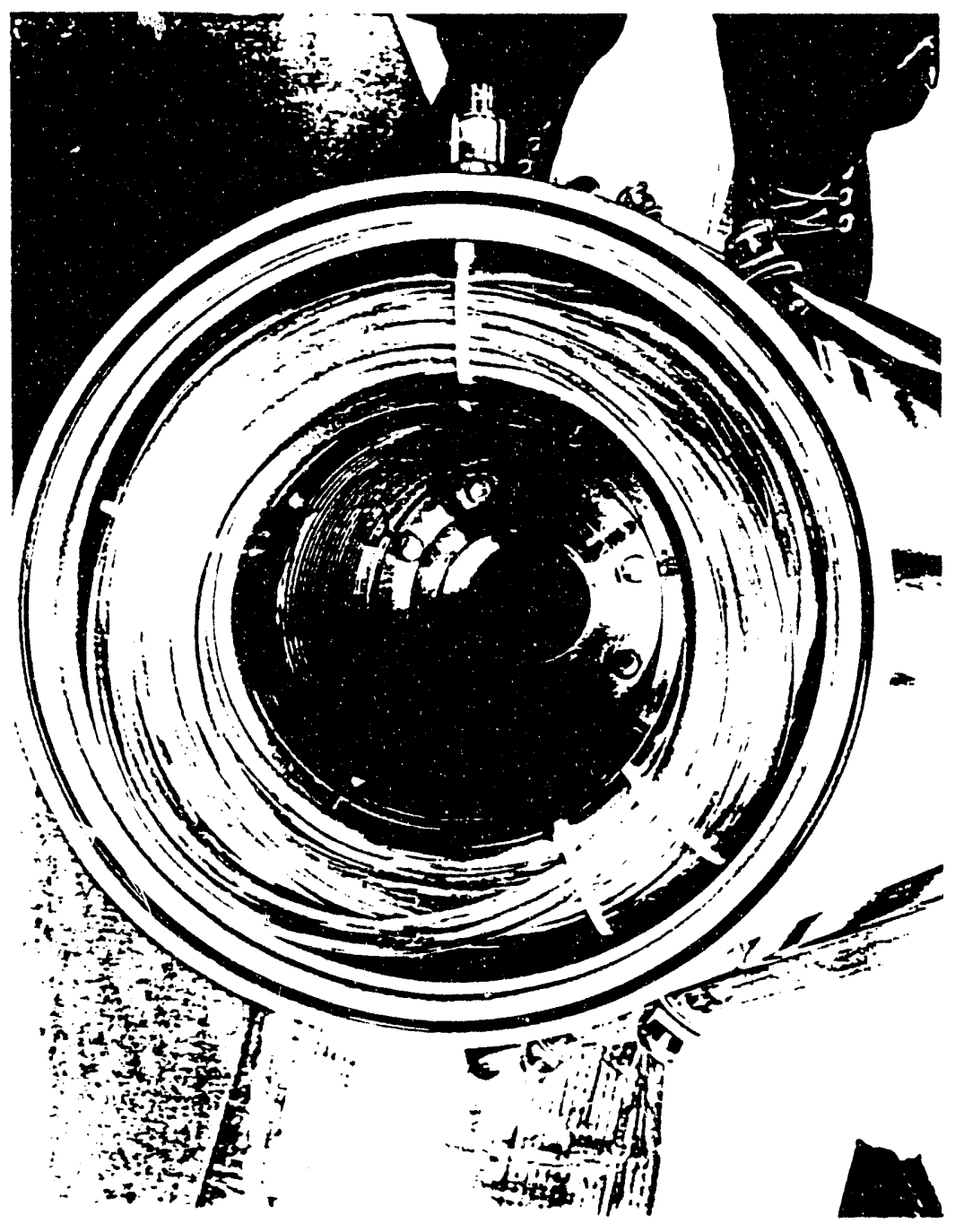

Figure 12. Picture of inducior core assembly and core reset coil. 
5. Shock Absorber - Tygon tubing, which is sealed at both ends, is wound to form a toroidal coil. This coil, which is located near the ground end of the $x$-ray tube, provides a trapped ait space in a defined location that serves to minimize pressure excursions associated with thermal expansions and with any shock waves created if an arc occurs.

6. Oil Eill Valves - Two valves are located on the end of the chamber opposite the $x$-ray tube for use in filling the chamber with oil. To fill the system, the top valve is connected to a mechanical vacuum pump, the chamber is evacuated, and then oil is introduced through the bottom valve.

7. Rogowski Coil - A Rogowski Coil is provided to measure the current in the $x$-ray tube. The calibration of this probe is $3.5 \mathrm{kA} / \mathrm{volt}$ when used with an integrator having $2 \mathrm{~ms}$ time constant.

8. Reset Circuit - This circuit, which is shown in Figure 13, is located within the HP cabinet. It provides $160 \mathrm{~V}$ at a peak pulsed current of 400 A with a fwhm pulse length of $\sim 2 \mathrm{~ms}$ at the reset connector on the chamber. The reset circuit is triggered at the same time the HP Marx is triggered so that the cores are reset within $2 \mathrm{~ms}$ after the high voltage pulse is delivered.

\section{ADDER OPERATION FITH THE HP43734A PUISER}

The equivalent circuit network for the pulser, adder, and $x-r a y$ tube is shown in Figure 14. As a result, the voltage $V_{L}$ applied across the $x$-ray tube is determined according to

$$
V_{L}=V_{m}\left[\left(R_{C} / 2\right) /\left(R_{m}+R_{C} / 2\right)\right]\left[R_{L} /\left(R_{C}+R_{I_{1}} / 2\right)\right]
$$




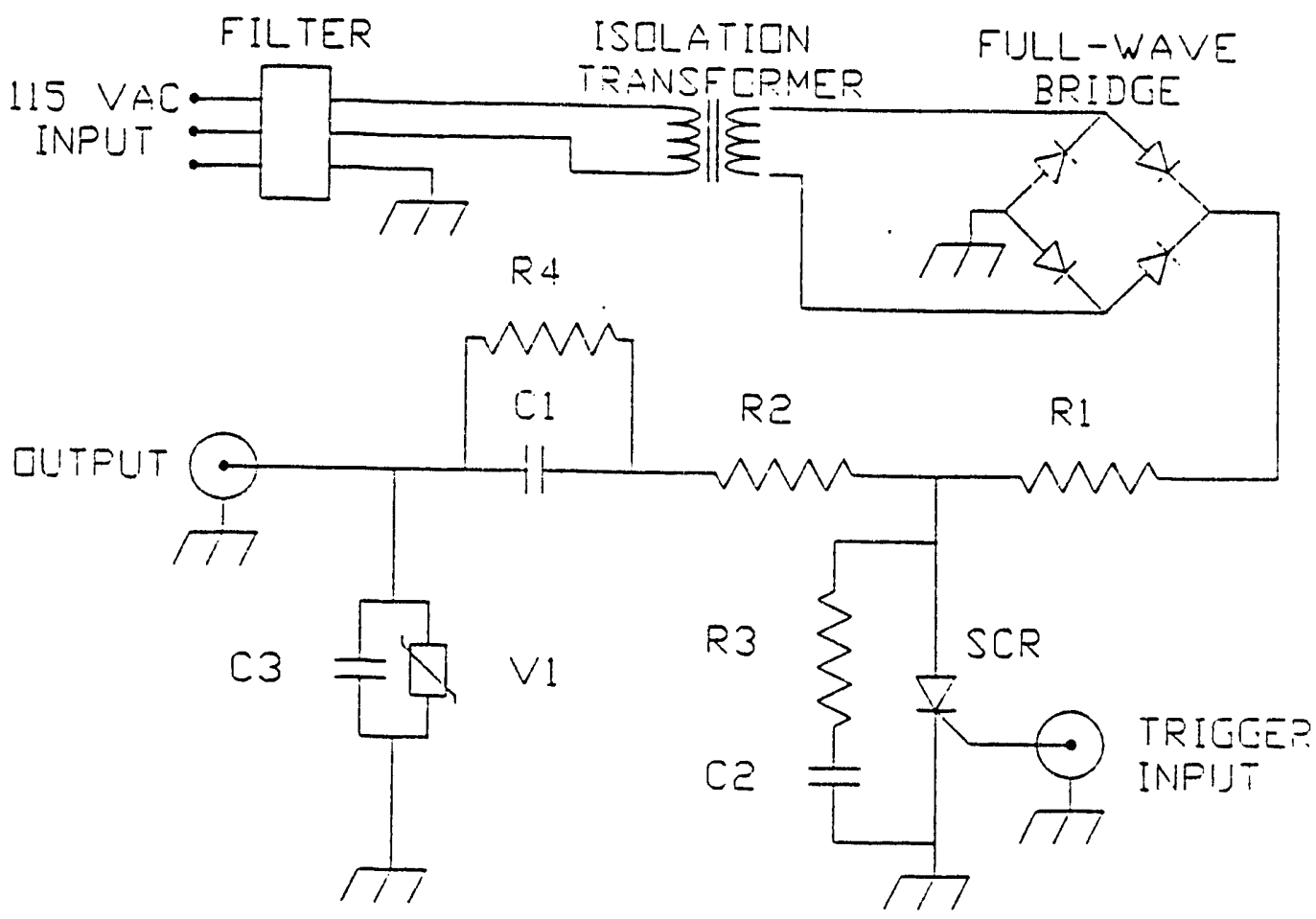

FILTER: COREOM \#6.J4

ISLLATION

TRANSFIRMER: TRIAD NG7A

FULL-WAVE

BRIDGE:

R1:

R2:

R3:

R 4:

$\mathrm{Cl}:$

C2:

C3:

SCR:

$\checkmark 1:$
IR, 200V', 10A

$1 \mathrm{~K}, 12 \mathrm{~W}$

2) .1 DHM, 5W, IN SERIES

10 DHM, $2 W$

100 KDHM, $2 W$

6) MALLORY HES IN PARALLEL, 2100UF, 400Y GE HI-F, IUF, 25OVAC

3) GE HI-F, IUF 250 VAC, IN PARALLEL

POWEREX C150D, 400V, 70A

GE C8, 120VAC

TRIGGER INPUT: BNC

QUTPUT: $\quad$ BNC

Figure 13. Reset circuit. 
where $R_{L}$ is the load impedance of the $x$-ray tube. The characteristic impedance $R_{C}$ of the high voltage cables is 63 ohms. Using resistive loads of varying impedance we separately determined that the internal impedance $R_{m}$ of the HP Marx pulser was about 85 ohms. The open circuit Marx voltage, $V_{m}$ is given by $V_{m}=30 V_{c h}$, where $v_{c h}$ is the charging voltage of the HP control console. Substituting these values into Eq. (23) yields

$$
V_{L}=16.2 V_{C h}\left[R_{L} /\left(63+R_{L} / 2\right)\right]
$$

which explicitly shows how the performance of the adder is determined by the impedance of the $x$-ray tube load.

\begin{tabular}{|c|c|}
\hline Marx Generator \\
\cline { 2 - 2 } Vo, Rm $=85$ onms & 63 ohms \\
\cline { 2 - 2 } & $\mathrm{RL} / 2\}$ \\
\end{tabular}

Eigure 14. Equivalent circuit of the Inductive Adder.

This predicted adder performance was subsequentiy checked by varying the impedance of a resistive load, which was inserted into the adder in place of the $x$-ray tube. The current through the resistive load was measured using calibrated B-dot and Rogowski coils. The peak output voltage data for several different charge voltages are displayed in Eigure 15 as a function of the impedance of the resistive load. These data are in substantial agreement with the performance predicted on the basis of Eq. (24), which is shown as the solid line. From these 
simple analyses it is apparent that substantially higher load voltages could be achieved if the impedance mismatch between the pulser and the two output cables were reduced.

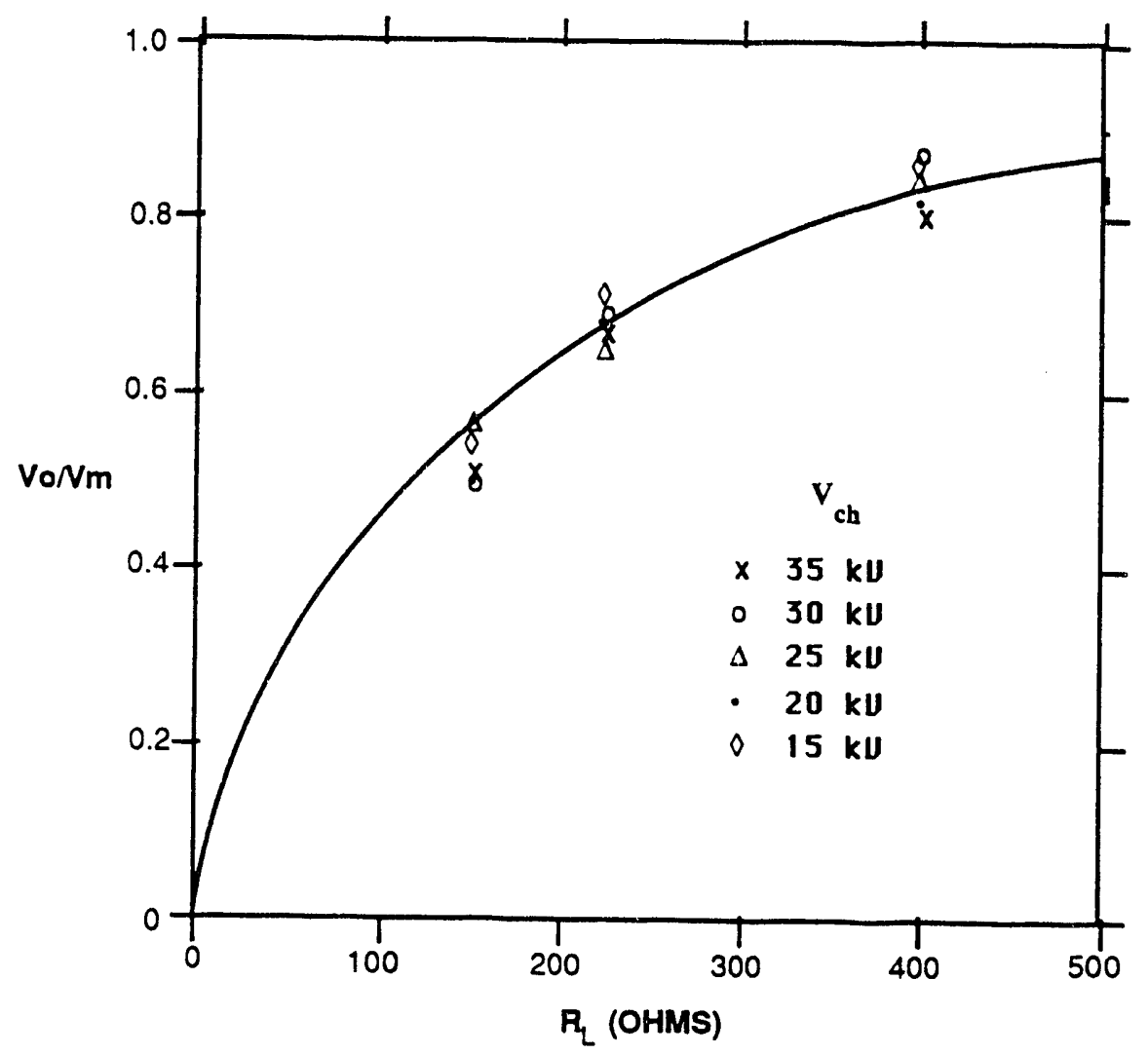

Figure 15. Dependance of load voltage on load impedance.

Following the resistive load checks a standard 1 MV HP tube (Model Number 5081-9551) was inserted into the adder. The current through the tube was monitored using a $B$-dot probe and a Rogowski coil, while the $x-$ ray pulse waveform was monitored using an uncalibrated PIN diode. The total $x$-ray dose was monitored using a quartz fiber dosimeter.

As an example of these data, Figure 16 shows the current through the standard $1 \mathrm{MV}$ tube for a charging voltage of $35 \mathrm{kV}$, while Figure 17 exhibits the output waveform of the $x$-ray pulse as measured by the PIN diode. The current waveform exhibits two distinct peaks, with the second being almost twice as large as the first. The total duration of this pulse is almost 90 ns. Although the RIN diode trace also indicates 

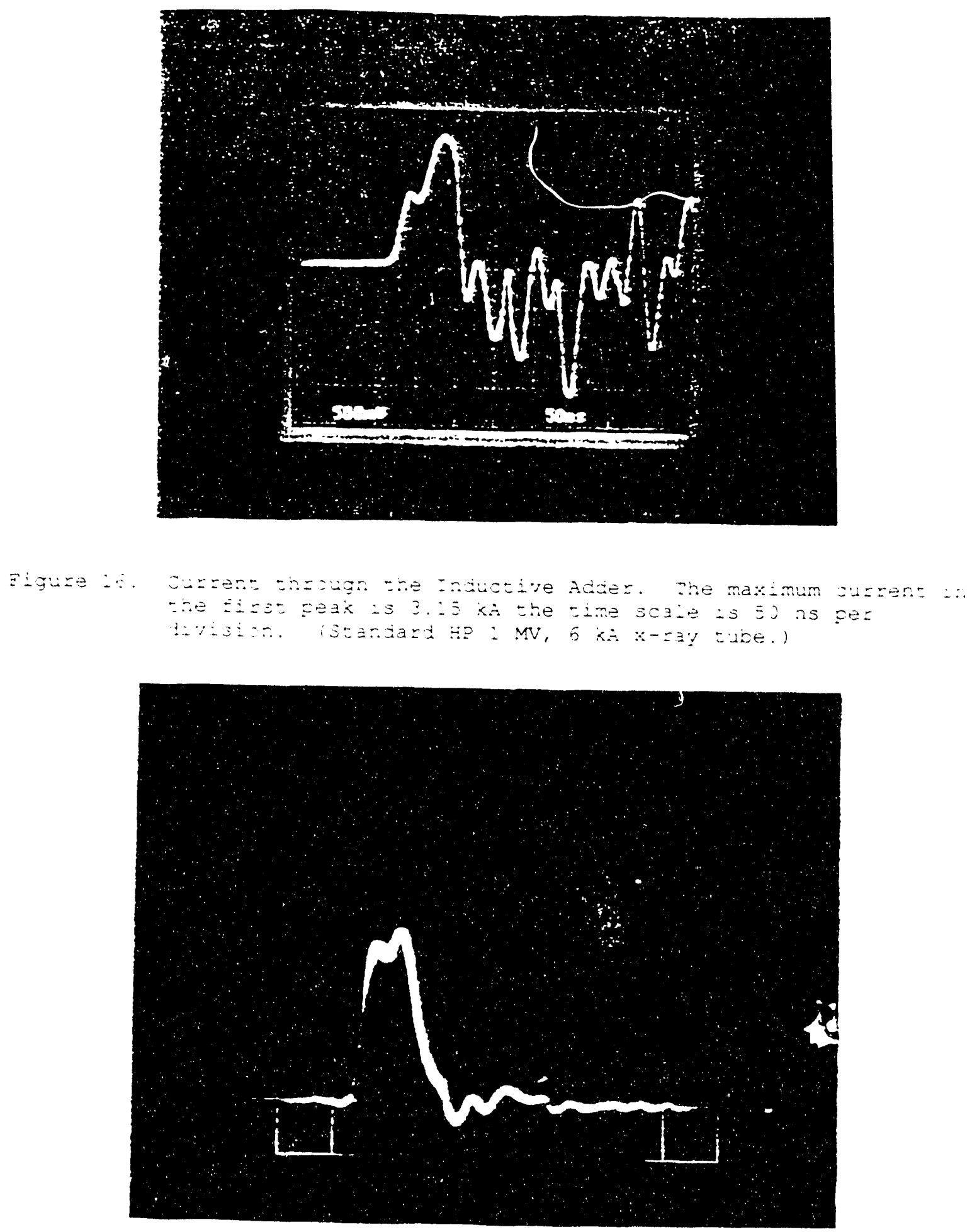

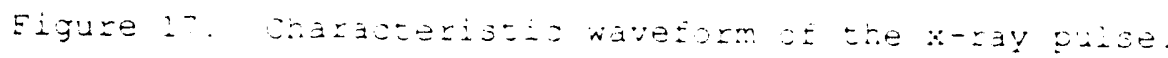


two peaks in the $x$-ray pulse, the second peak is approximately equal to the first peak in amplitude, and the FWHM of the x-ray pulse is about 50 ns.

These data suggest that the $\mathrm{x}$-ray tube impedance collapses as the result of plasma closure in the anode-cathode gap of the tube at late times in the pulse. Assuming the correctness of our circuit model, the amplitude of the first current peak ( $3.15 \mathrm{kA}$ ) implies a nominal tube impedance of 234 ohms, and the corresponding output voltage across the tube is $737 \mathrm{kV}$. In comparison, the amplitude of the second current peak (6.3 kA) implies that the tube impedance has decreased to 54 ohms, and the tube voltage has dropped to $340 \mathrm{kV}$. The load impedances and tube voltages corresponding to the first current peak are graphed as a function of the Marx charging voltage in Figures 18 and 19.

The total x-ray dose as measured by the quartz fiber dosimeter located one meter from the tube is also displayed in Figure 20 as a function of Marx charging voltage. A maximum dose of $30 \pm 5 \mathrm{mR}$ is obtained at the highest charging voltage ( $35 \mathrm{kV})$, although the peak voltage on the $\mathrm{x}$-ray tube apparently never exceeds about $740 \mathrm{kV}$.

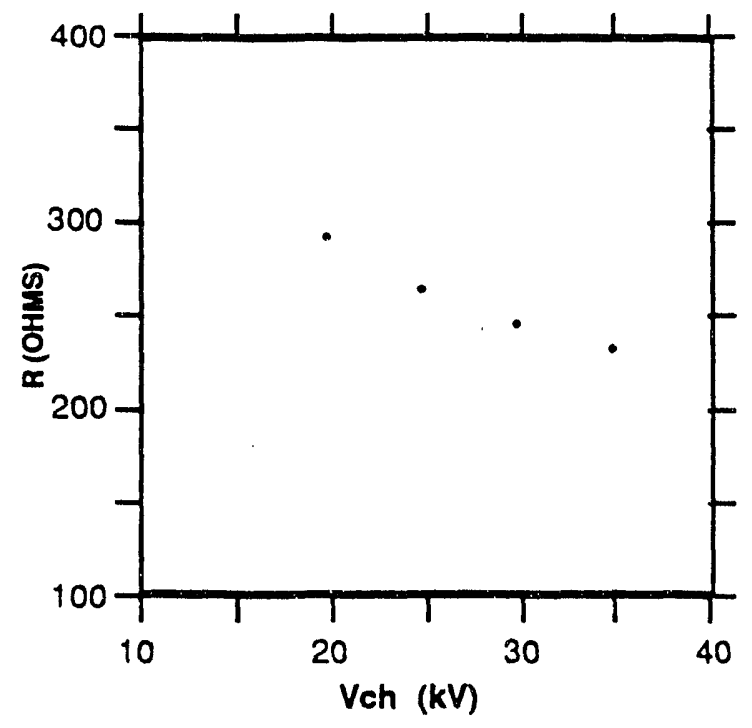

Eigure 18. Load impedance of the standard $1 \mathrm{MV} x$-ray tube as a function of Marx charge voltage. 


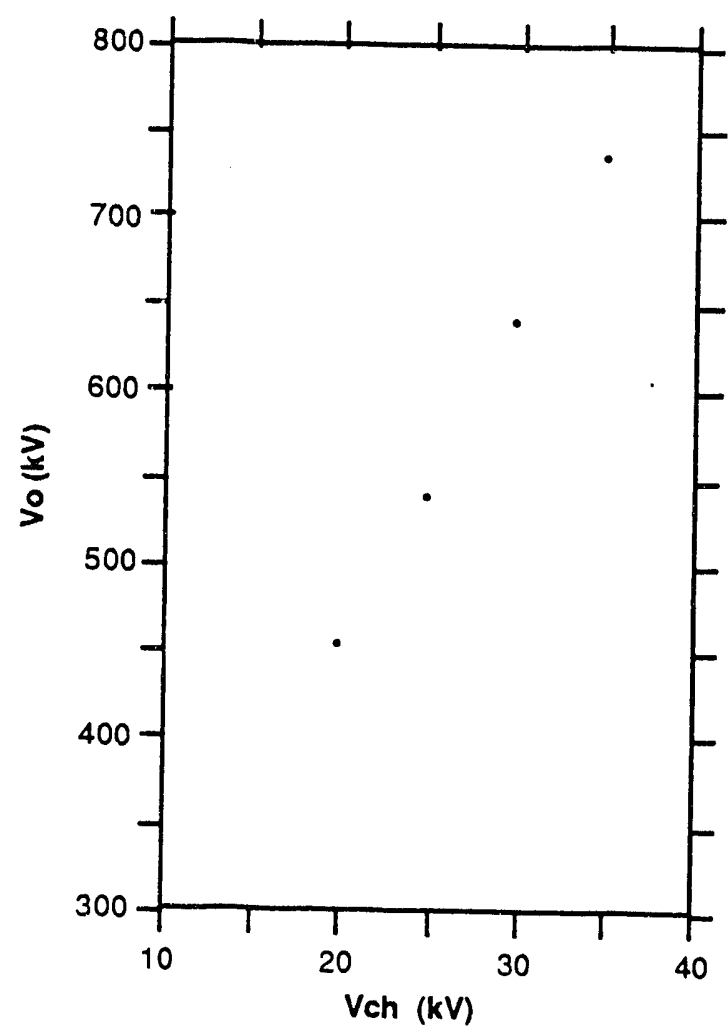

Figure 19. Adder output voltage for the standard $1 \mathrm{MV} \mathbf{x}$-ray tube as a function of Marx charge voltage.

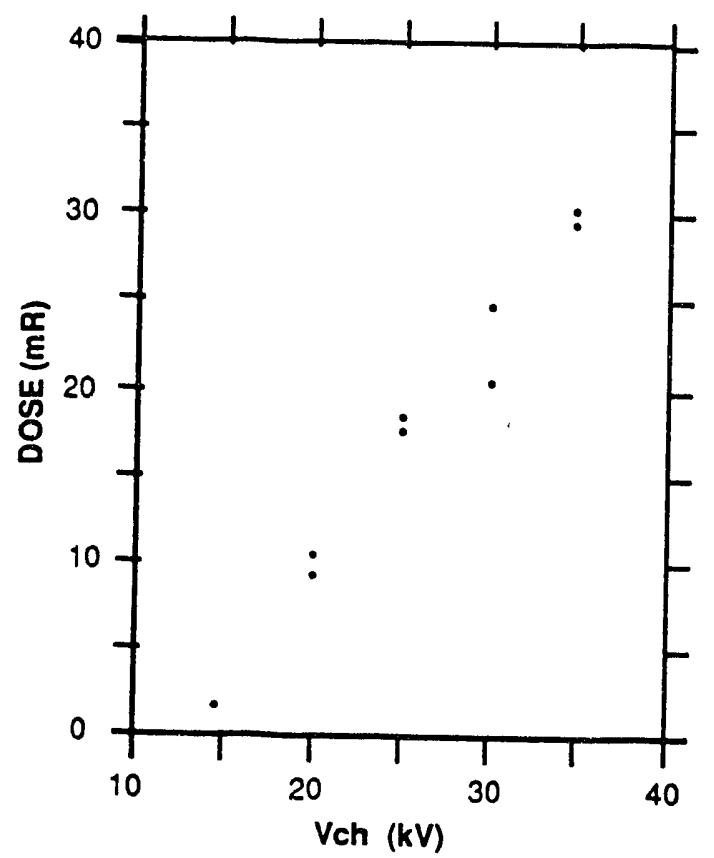

Figure 20. X-ray dose at one meter vs Marx charge voltage (standard 1
MV tube). 
The standard HP 1 MV tube has six cathode splines. In an effort to increase the tube impedance (thus allowing a higher voltage across the tube), a three-spline tube was specially ordered. The resulting adder current trace for a charging voltage of $35 \mathrm{kV}$ is shown in Figure 21. The first peak of the current pulse is now $2.6 \mathrm{kA}$, and the average dose at one meter has increased to $35 \pm 5 \mathrm{mR}$. The corresponding tube impedance is 310 ohms, and the peak calculated voltage is $806 \mathrm{kV}$. These values are essentialiy equal to the requirements for the Los Alamos radiograpicic machine. Impedance values and peak voltages across the new high impedance tube are graphed in Figures 22 and 23 as a function of Marx charging voltage.

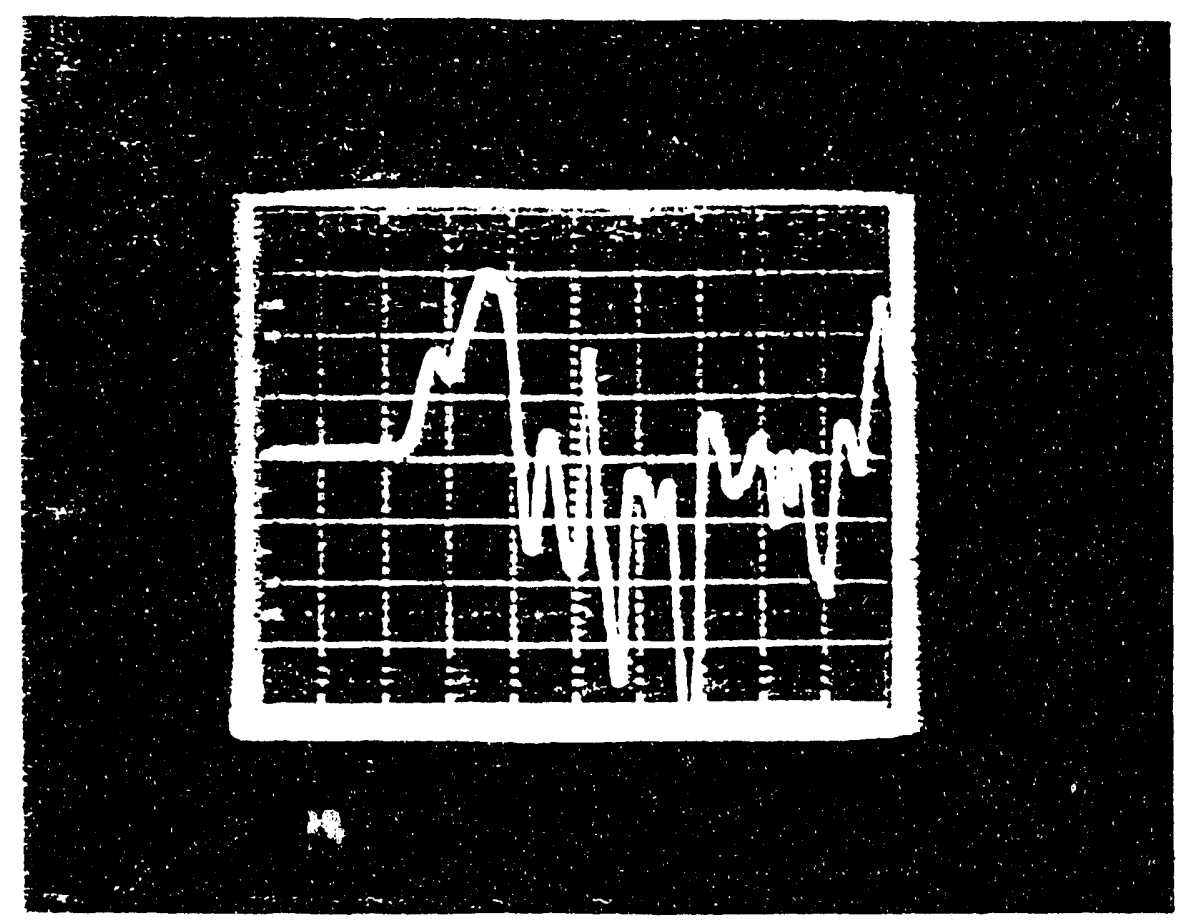

Figure 21. Current through the Inductive Adder. The maximum current in the first peak is $2.62 \mathrm{kA}$. (Modified high impedance HP $x$-ray tube.) 


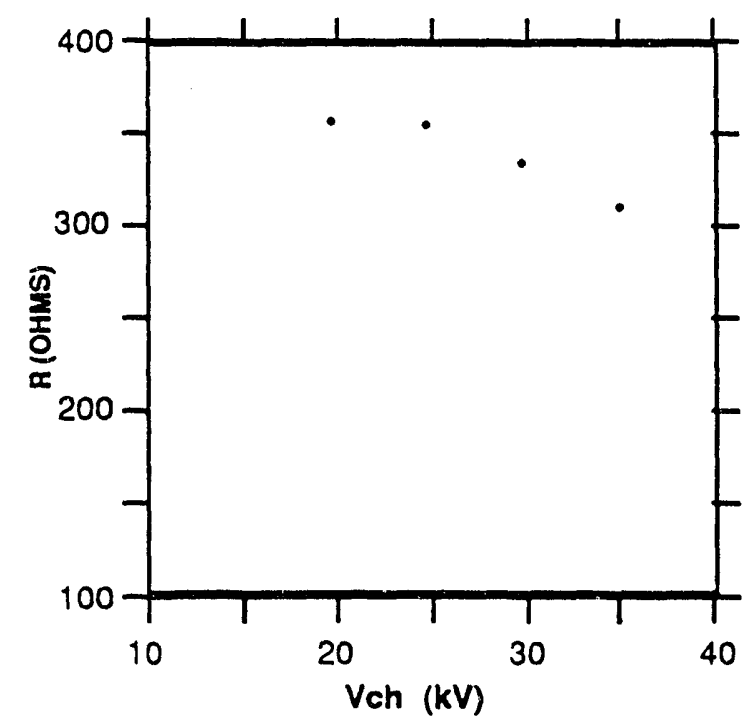

Eigure 22. Load impedance of the modified high impedance 1 MV tube as a function of Marx charge voltage.

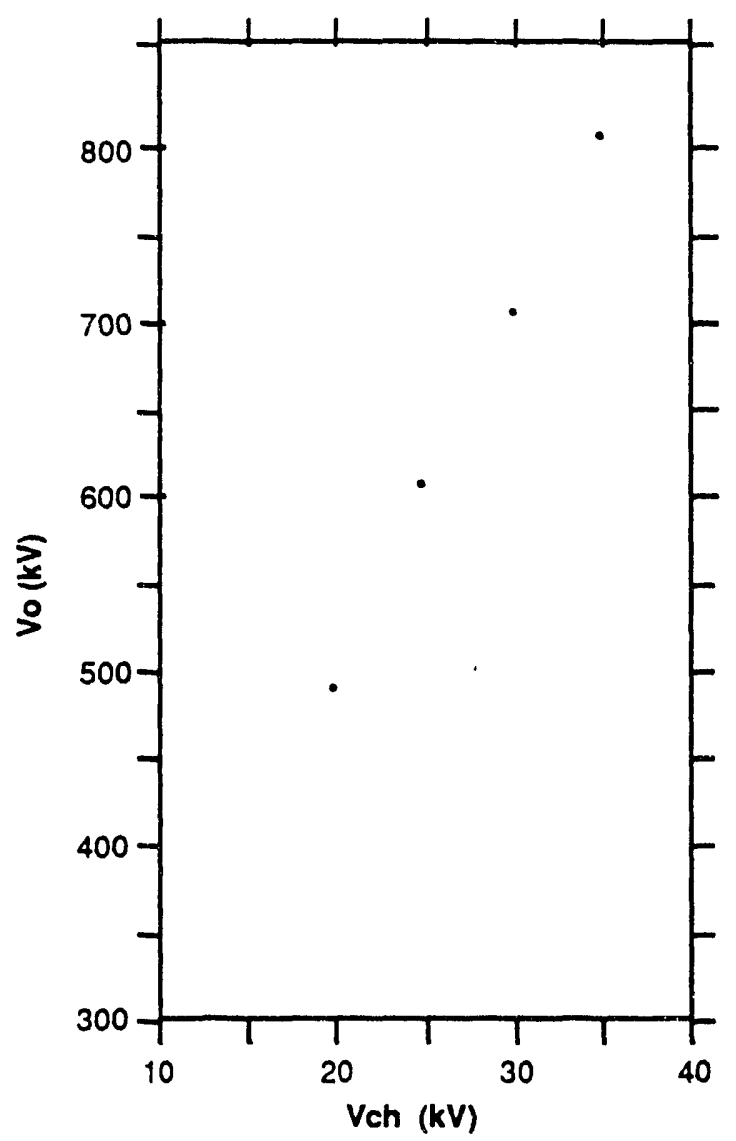

Eigure 23. Adder output voltage for the modified high impedance 1 MV tube as a function of Marx charge voltage. 
VIII. X-RADIOGRAPHY EXPERIMENTS USING STATIONARY TARGETS

In order to demonstrate the enhanced penetrating power provided by the inductive adder, we performed several radiography experiments using stationary targets. Prior to these measurements we first determined the decrease in $x$-ray dose delivered to a quartz fiber dosimeter placed onehalf meter from the high impedance tube as a function of Marx charging voltage. These data are graphed in Figure 24. Very roughiy, tine thickness of steel required to reduce the dose by a factor of 2 was about 0.25 inches.

The configuration for the radiography tests is shown in Figure 25 . The target set could be placed between various thicknesses of steel plate, and the tube-target and target-film plane distances could be varied independently. The target set consisted of six steel ball bearings with diameters of $0.1250 \mathrm{in,} 0.1555 \mathrm{in}, 0.1875 \mathrm{in}, 0.2505 \mathrm{in}$, $0.3155 \mathrm{in}$, and $0.3765 \mathrm{in}$, respectively. A small lead shot pellet of 0.0806 in $(3.17 \mathrm{~mm})$ diameter was an additional object.

The $x$-ray film used in these measurements was Kodak XAR-7 x-ray film, kindly provided by Mr. Jake Lucero of LANL's M-8 Group. In addition, we used three different image intensifier screens, a CRONEX NDT-2, NDT-9, and a CRONEX QUANTA FAST DETAIL screen, which were loaned to TITAN by the Air Force Weapons Laboratory.

A complete list of all $x$-ray tests is included in Table 1 . Within. the limits of our film reproducing capabilities, several examples are exhibited in Eigures 26-31. As a general summary, we could easily record all seven target objects through an inch of steel plate. In fact, we were able to record very good target images through up to 1.75 inches of steel. We obtained best results with the NDT-2 image intensifier screen. In addition, use of a 0.25 in thick lead aperture (to reduce scattering from the steel plate, as shown in Figure 3 ) also improved our target images. Best results were generally obtained at the highest charging voltages, especially for the thickest layers of 
absorber. However, by varying the time for film developing and using thicker image intensifier screens we were able to obtain very good images at lower voltages for smaller thicknesses of absorber. As expected, the smallest target object, the lead pellet, was easier to observe than the steel ball bearing which was almost twice as thick.

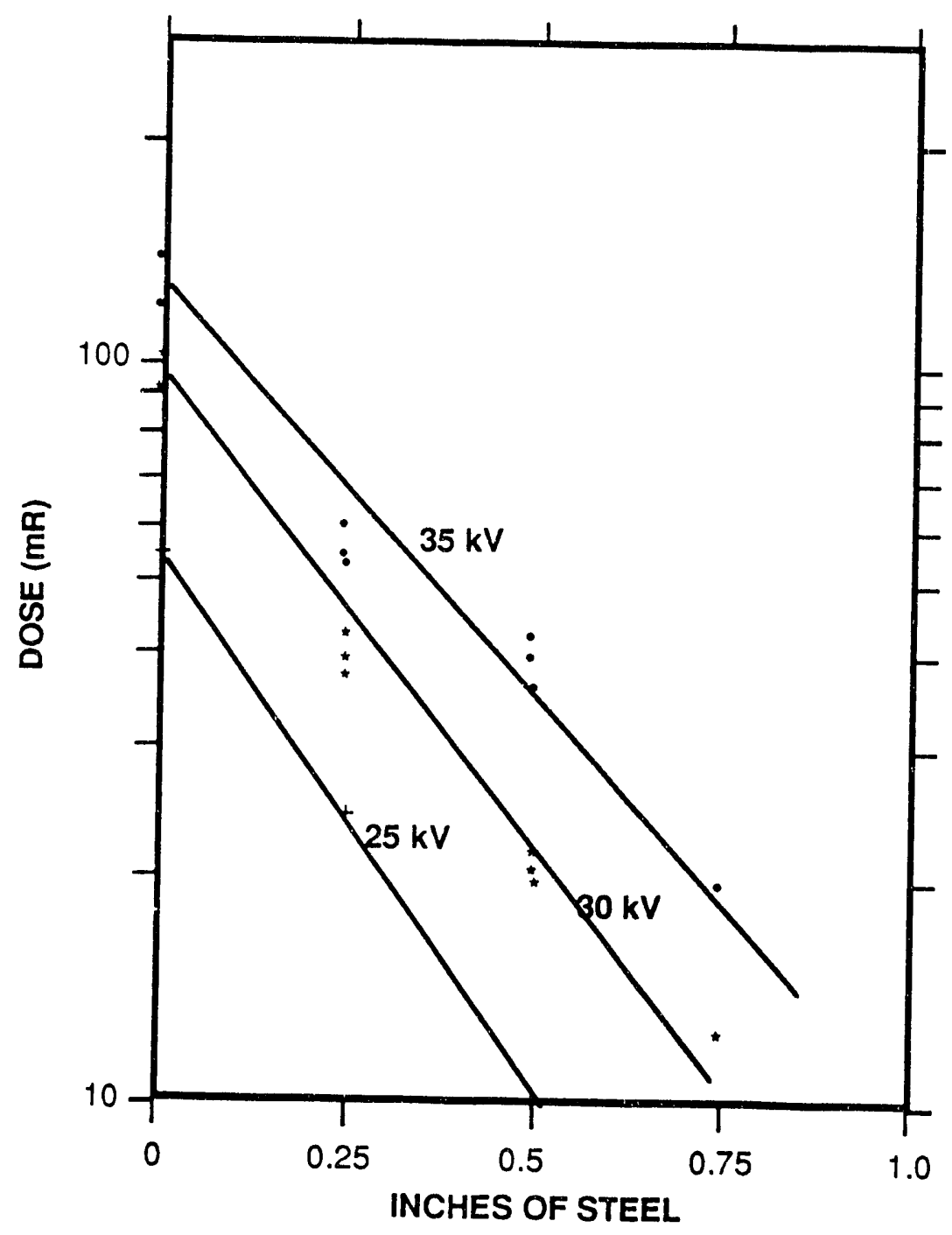

Figure 24. Variation of $x$-ray dose at $50 \mathrm{~cm}$ from the tube as a function of steel penetration thickness for several Marx charge
voltages. 


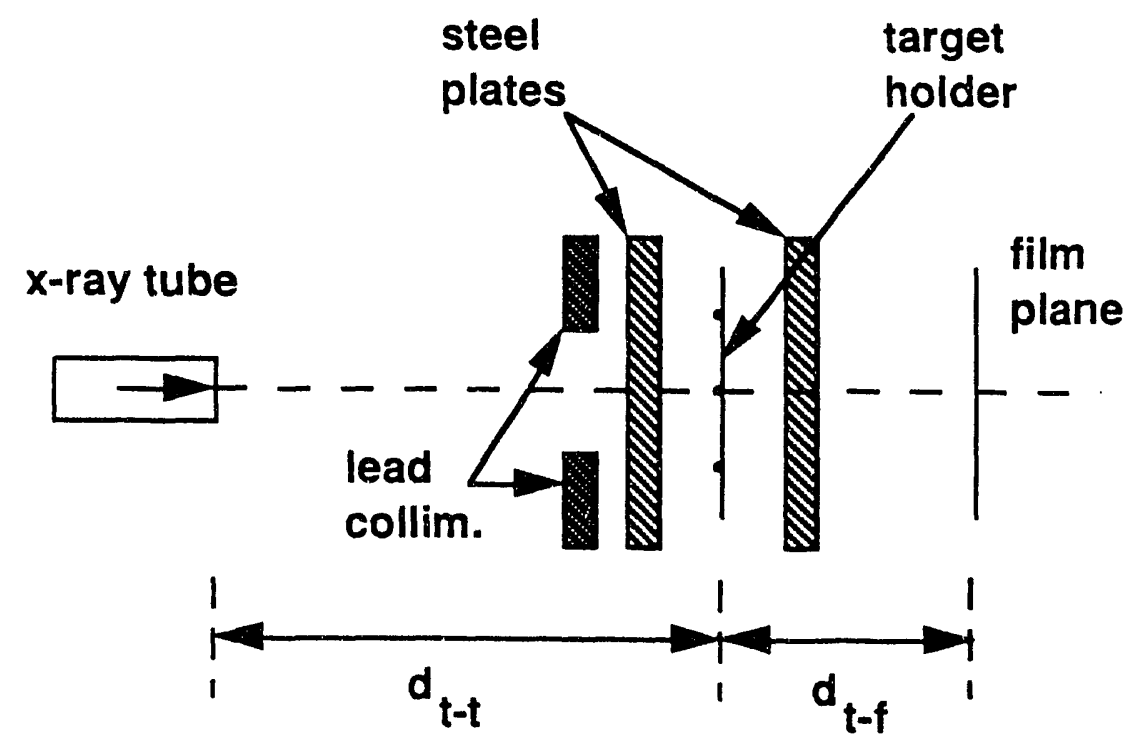

Figure 25. Configuration of radiography tests.

Based on these observations, use of the inductive adder in conjunction with the HP $43734 \mathrm{~A}$ pulser should permit the observation of few-millimeter-thick tungsten (or other suitable high-z/high-density material) shards through an inch of steel armoring, provided that the tube-film plane distance is less than about one meter. Some experimentation with different recording films, developer times, image intensifiers, etc. is recommended to obtain best results. 


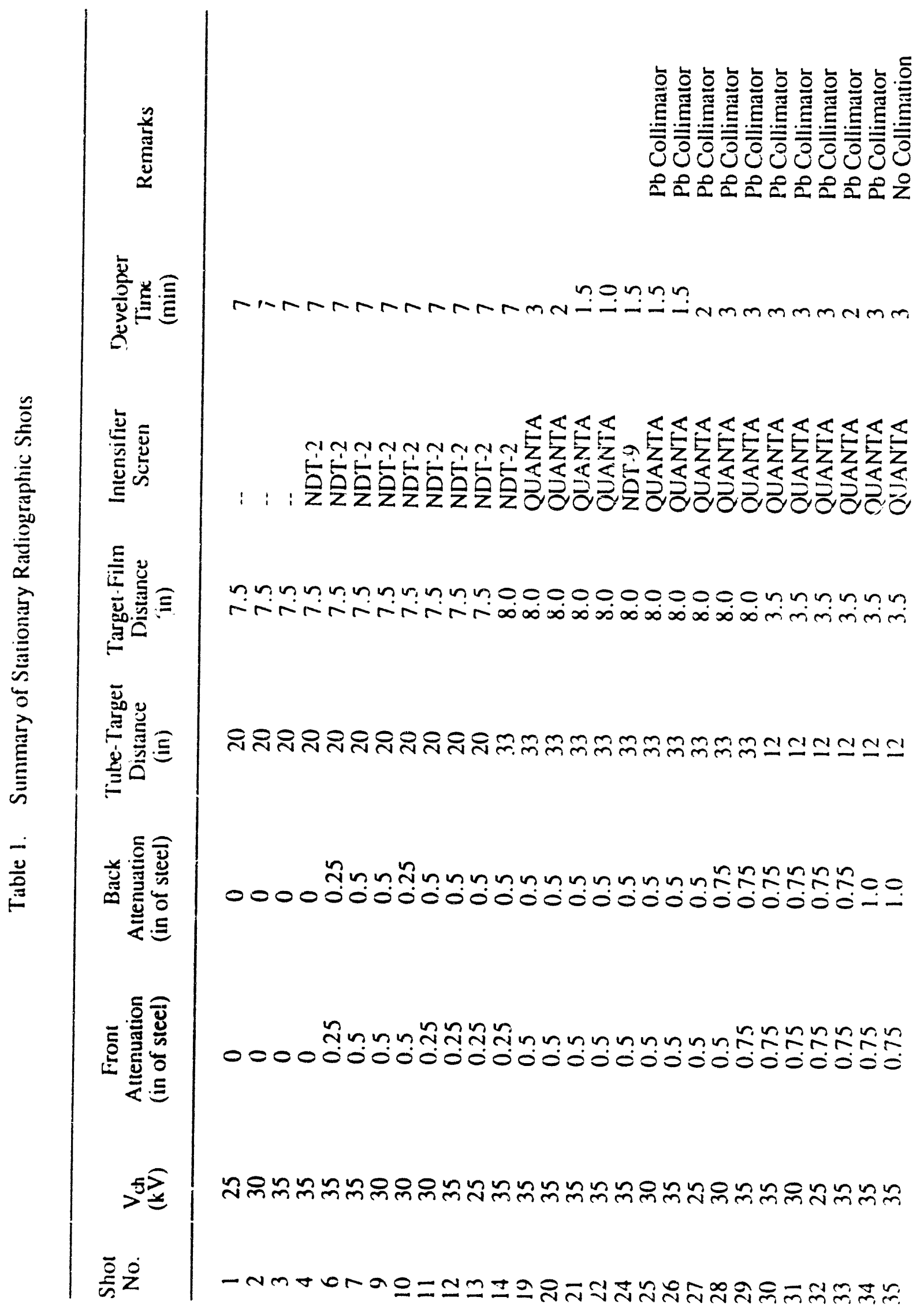




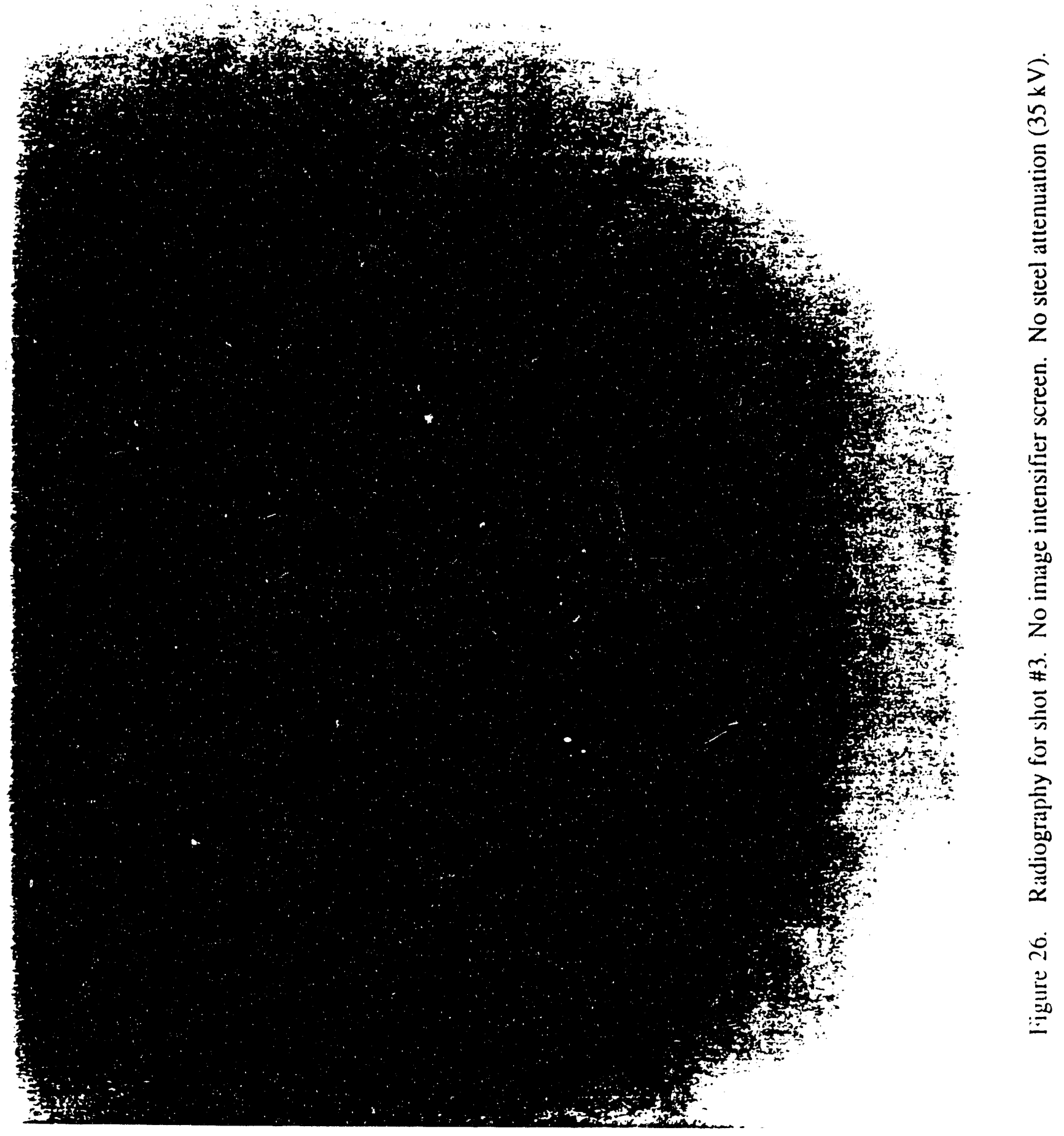




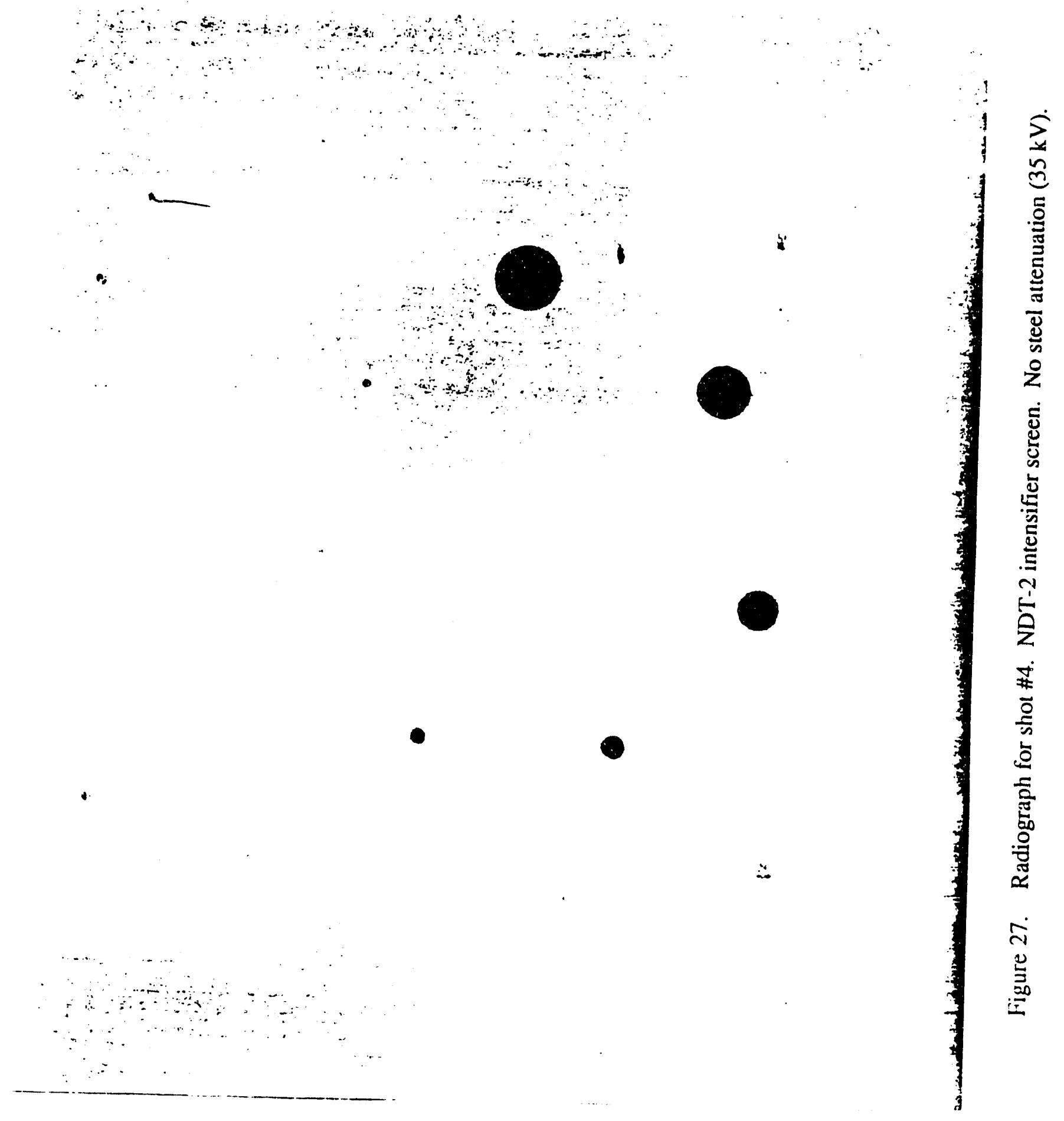




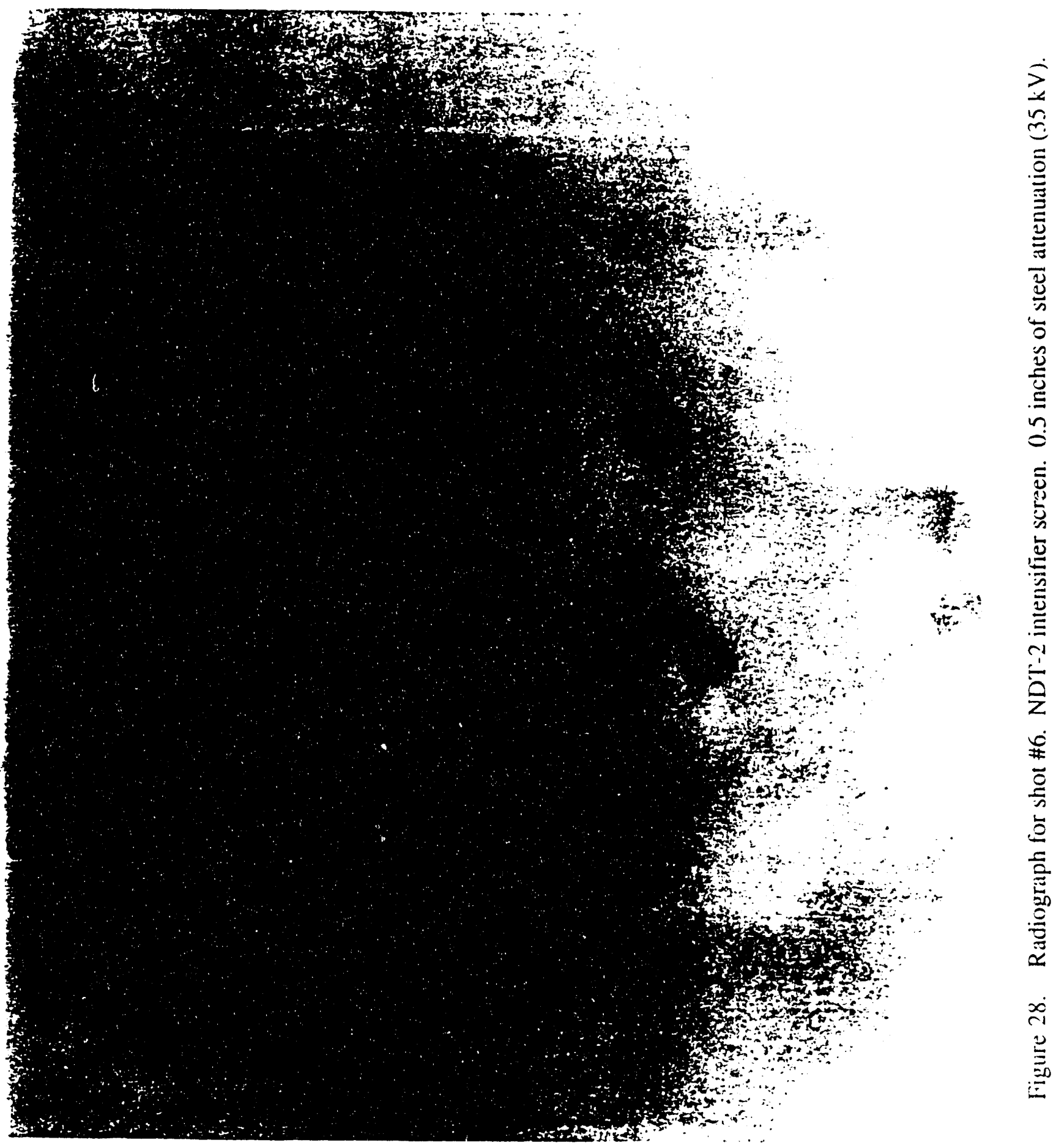




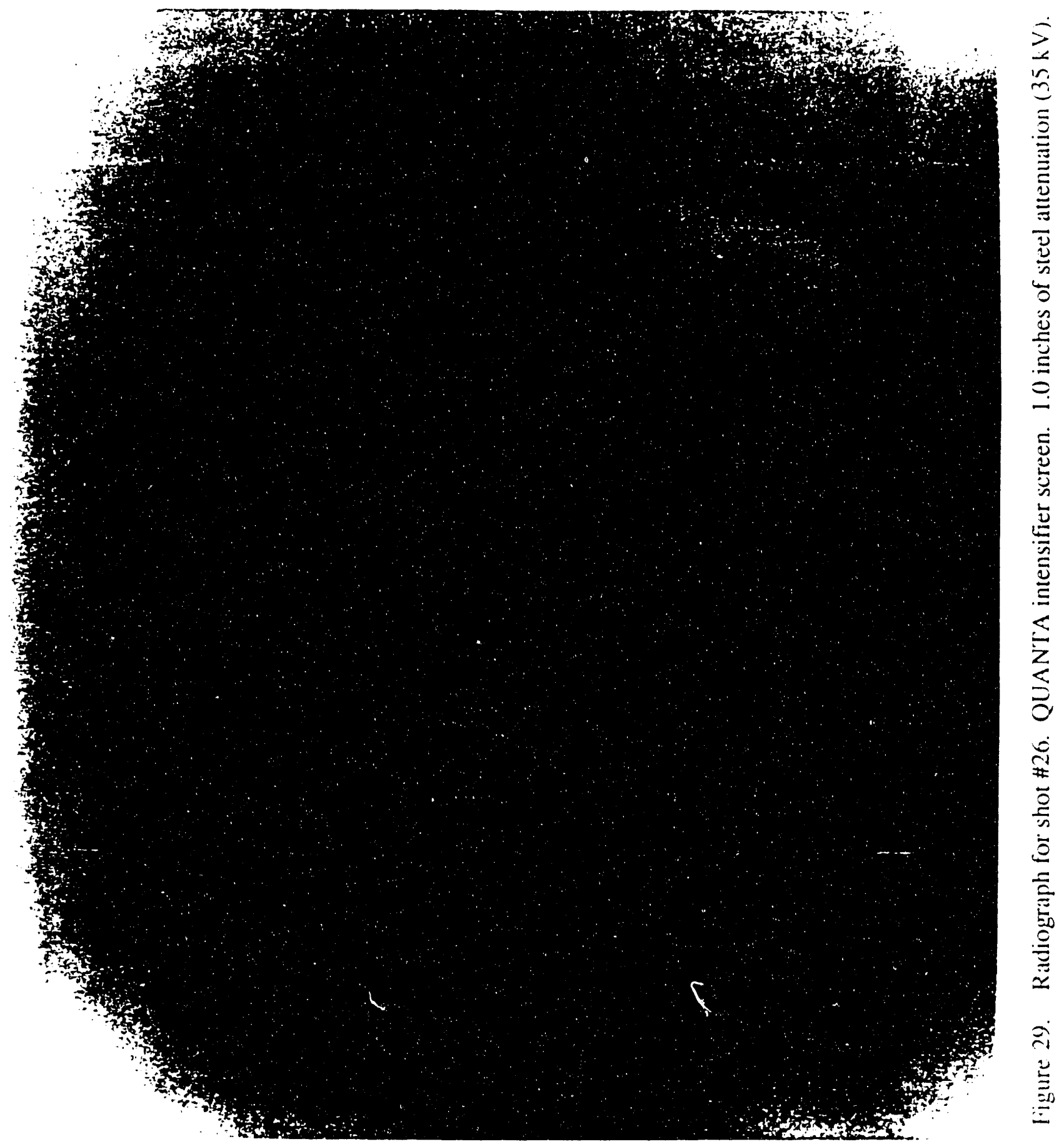




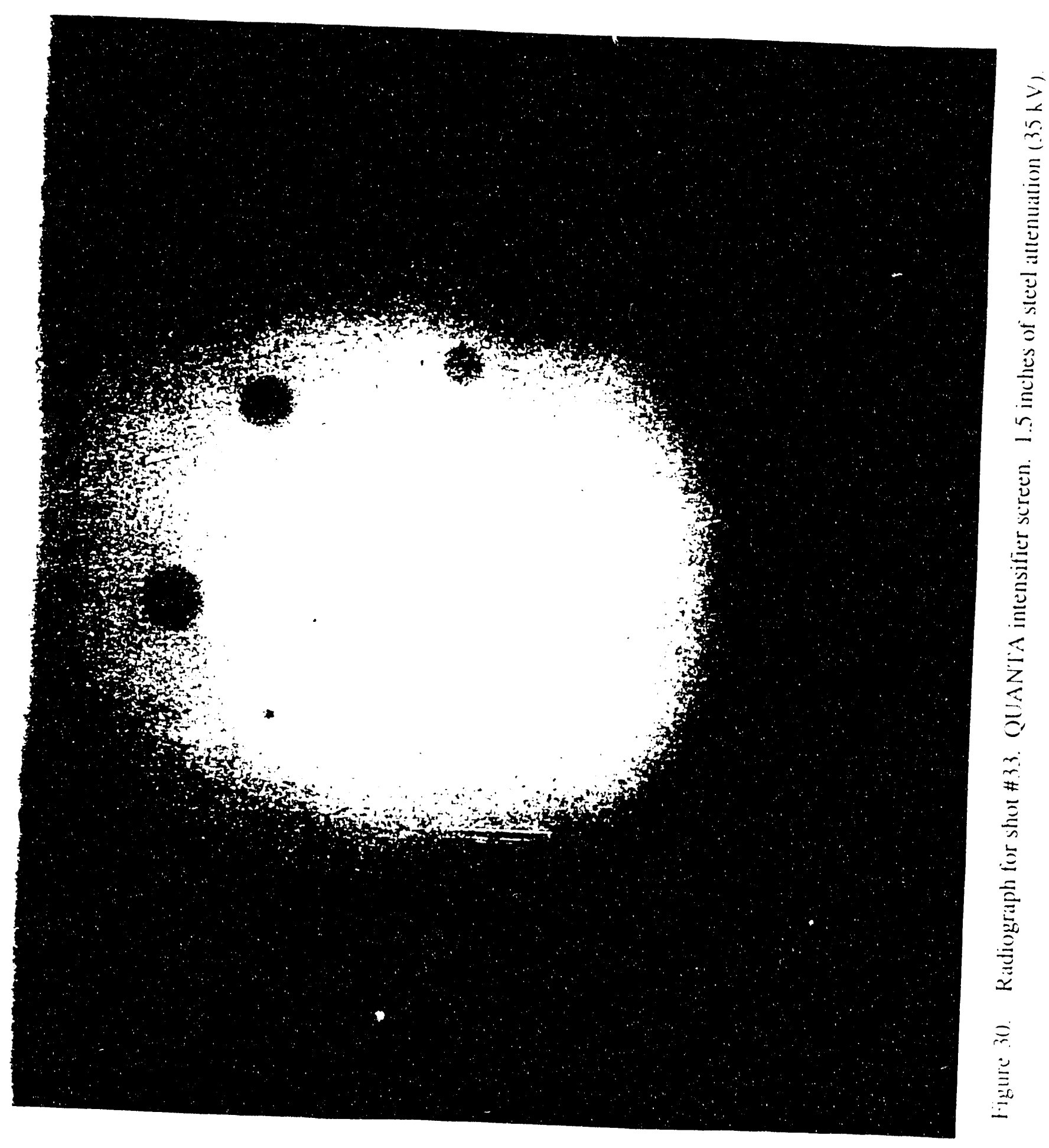


(1) 4
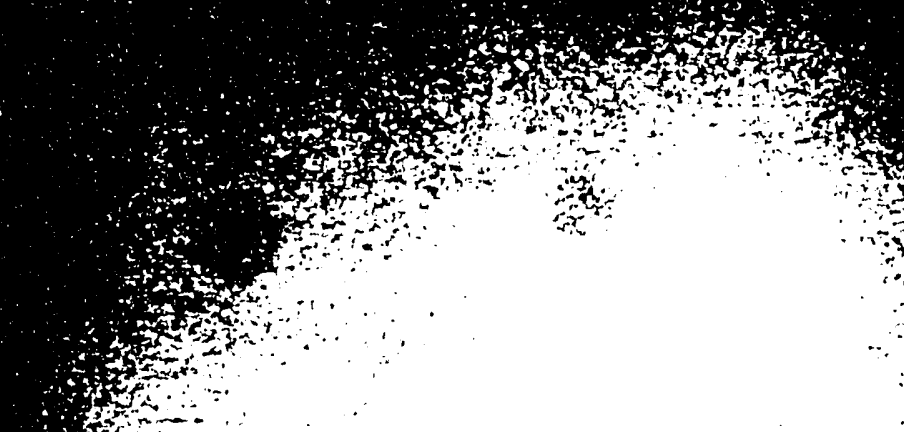

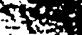


IX . SUMMARY

TITAN has successfully developed an inductive adder for use with a Hewlett-Packard Model 43734A Marx pulser. The unit provides an $800 \mathrm{kV}$ peak output pulse to a modified HP 5081-9551 $1 \mathrm{MV}$ x-ray tube. The tube fits into the adder unit, and can thus be remotely operated. The total on-axis $x$-ray dose at one meter from the tube is $35 \pm 5 \mathrm{mR}$, as measured by a quartz fiber dosimeter. The EWHM duration of the radiation pulse is $\leq 50 \mathrm{~ns}$. Based on the the results of radiography measurements using stationary targets, this unit permits the user to observe fewmillimeter-thick tungsten for other suitable high-z/high-density material) shards through an inch of steel armoring, provided that the tube-film plane distance is less than about one meter.

Although not demonstrated here, the special construction of the adder should permit multiple pulse operation at approximately $350 \mathrm{kV}$, provided that catastrophic plasma closure does not occur in the $x$-ray tube between $x$-ray pulses. It should also be possible to repeat the adder process more than once. Finally, a single high voltage cable output from the Marx generator (with a splitting inside the adder unit) would decrease the impedance mismatch and further increase the voltage by approximately $15 \%$. 
X . REFERENCES

1. For a similar treatment see W.R. Van Antwerp and W.B. Beverly, "Studies on Ballistic Application of Bremsstrahlung Radiography," BRL-TR-2660, (June, 1985).

2. E. Segre, Nuclei and Particles, W.A. Benjamin, New York, (1965).

3. J.C. Golden, "An Analytical Expression for Bremsstrahlung High Eiltered, Thick High-Z Target, 2-20 MeV," AEC-SC-DC-67-2339, (April, 1968).

4. T.H. Martin, "Determination of Bremsstrahlung Production Efficiencies from Experimental Data at $27 \mathrm{MeV}, "$ Bull. Am. Phys. Soc. 13, 1406 (1968).

5. D.R. Goosman, "Some Aspects of Thick-Object Radiography," Ad Hoc Hydrodiagnostics Exchange Meeting (AVIS 075), LLNI (October, 1976).

6. J.H. Hubbell, "Photon Cross Sections, Attenuation Coefficients, and Energy Absorption Coefficients from $10 \mathrm{keV}$ to $100 \mathrm{GeV}$, "NSRDSNBS 29, (August, 1969).

7. J.A. Halblieb and W.H. Vandevender, 'TIGER: A One-Dimensional, Multi-Layer Electron/Photon Monte Carlo Transport Code," SLA-731926, (March, 1974). 

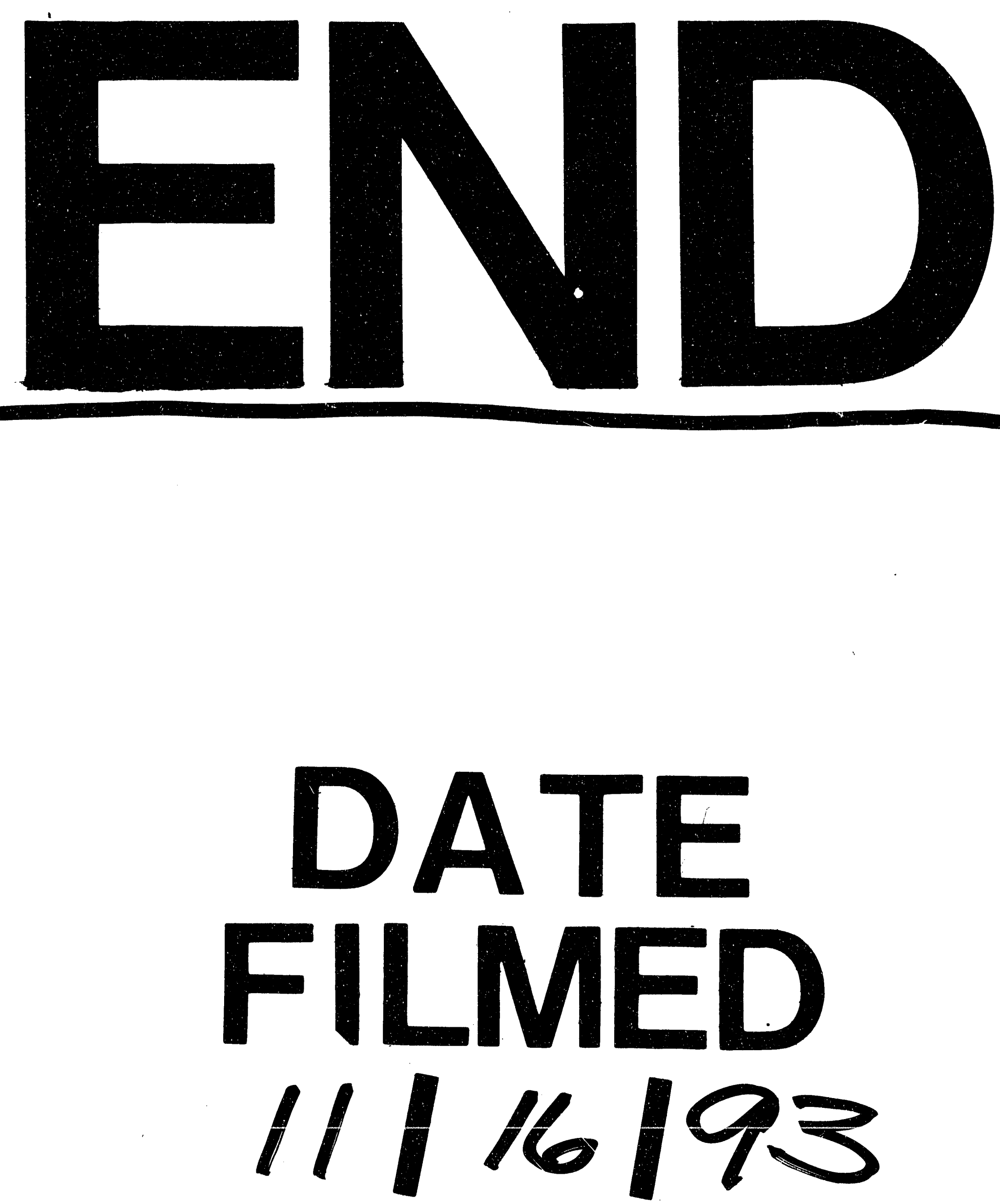
